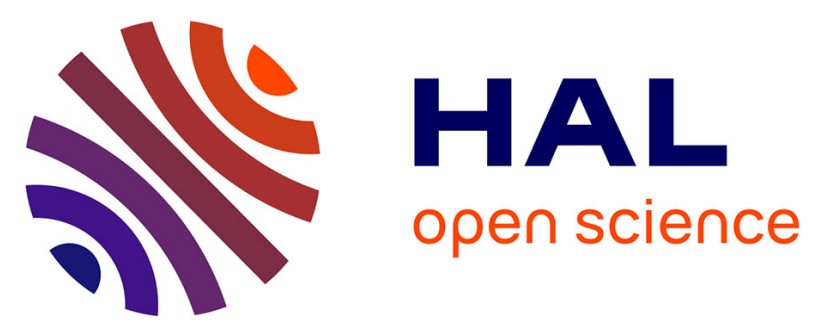

\title{
Sequential identification of boundary support parameters in a fluid-structure vascular model using patient image data
}

Philippe Moireau, Cristobal Bertoglio, Nan Xiao, C. Alberto Figueroa, Charles Taylor, Dominique Chapelle, Jean-Frédéric Gerbeau

\section{To cite this version:}

Philippe Moireau, Cristobal Bertoglio, Nan Xiao, C. Alberto Figueroa, Charles Taylor, et al.. Sequential identification of boundary support parameters in a fluid-structure vascular model using patient image data. Biomechanics and Modeling in Mechanobiology, 2012, 12 (3), pp.475-496. 10.1007/s10237012-0418-3 . hal-00760703

\section{HAL Id: hal-00760703 https://hal.inria.fr/hal-00760703}

Submitted on 4 Dec 2012

HAL is a multi-disciplinary open access archive for the deposit and dissemination of scientific research documents, whether they are published or not. The documents may come from teaching and research institutions in France or abroad, or from public or private research centers.
L'archive ouverte pluridisciplinaire HAL, est destinée au dépôt et à la diffusion de documents scientifiques de niveau recherche, publiés ou non, émanant des établissements d'enseignement et de recherche français ou étrangers, des laboratoires publics ou privés. 


\title{
Sequential identification of boundary support parameters in a fluid-structure vascular model using patient image data
}

\author{
P. Moireau - C. Bertoglio - N. Xiao - C.A. Figueroa - C.A. Taylor • \\ D. Chapelle • J-F. Gerbeau
}

Received: 15/05/2012 / Accepted: date

\begin{abstract}
Visco-elastic support has been previously established as a valuable modeling ingredient to represent the effect of surrounding tissues and organs in a fluidstructure vascular model. In this paper, we propose a complete methodological chain for the identification of the corresponding boundary support parameters, using patient image data. We consider distance maps of model to image contours as the discrepancy driving the data assimilation approach, which then relies on a combination of (1) state estimation based on the socalled SDF filtering method, designed within the realm of Luenberger observers and well-adapted to handling measurements provided by image sequences, and (2) parameter estimation based on a reduced-order UKF filtering method which has no need for tangent operator computations and features natural parallelism to a high degree. Implementation issues are discussed, and we show that the resulting computational effectiveness of the complete estimation chain is comparable to that of a direct simulation. Furthermore, we demonstrate the use of this framework in a realistic application case involving hemodynamics in the thoracic aorta. The estimation of the boundary support parameters proves successful, in particular in that direct modeling simulations based on the estimated parameters are more accurate than with a previous manual expert calibration. This paves the way for complete patient-specific
\end{abstract}

P. Moireau · C. Bertoglio - D. Chapelle - J-F. Gerbeau Inria, Rocquencourt B.P.105, 78153 Le Chesnay, France

N. Xiao · C.A. Figueroa

Department of Biomedical Engineering, King's College London, London SE1 7EH, United Kingdom

N. Xiao · C.A. Taylor

Department of Bioengineering, Stanford University, Clark Center, 318 Campus Drive, Stanford, CA 94305-5431, United States fluid-structure vascular modeling in which all types of available measurements could be used to estimate additional uncertain parameters of biophysical and clinical relevance.

\section{Introduction}

In the past decade, numerous works have broached the topic of fluid-structure interaction (FSI) in arteries, with an increasing focus on the concept of patient-specific modeling. This polysemic term was initially mainly associated with the anatomical personalization of the modeled arteries, which justified major efforts to improve segmentation techniques in order to produce patientspecific geometries (Cebral et al 2005; Gerbeau et al 2005; Alastrué et al 2010; Xiong et al 2010). In addition, many modeling specific components have been incorporated into the FSI formulation to improve the representation of the patient flow characteristics. In particular, a significant emphasis has been placed on fluid boundary conditions - see for example (Figueroa et al 2006; Formaggia et al 2001; Kerckhoffs et al 2007; Quarteroni et al 2001) - to model the impact of the part of the arterial tree truncated from the region of interest. Given the complexity of the truncated arterial tree, it is common to substitute it with simplified lumped parameter models at the inlet and outlets, specifically calibrated with the available pressure and flow data available for the patient (see for example (Brown et al 2011; Troianowski et al 2011)).

With recent advances in medical imaging technology such as cardiac-gated Computed Tomography (CT) and 4D Magnetic Resonance Imaging (MRI) that can now provide time-resolved data on the motion of the arterial wall over the cardiac cycle, a new wealth of in- 
formation is available to inform the simulation efforts still further. An approach - sometimes called "imagebased CFD", see e.g. (Moreno et al 2006; Piccinelli et al 2010) - has been proposed whereby the flow is computed in a domain whose deformation is directly deduced from the medical images, thus avoiding the resolution of the coupled fluid-solid problem. However, this type of approach features important limitations in that inaccuracies inherent to such measurements then directly produce significant error sources in the modeling simulations, and some important physical phenomena intrinsic to FSI - such as pressure waves - are not adequately captured. By contrast, a different approach has been adopted in some cardiac and arterial FSI applications (Chabiniok et al 2011; Bertoglio et al 2012b), using the time-resolved image data extracted from images and a cardiac mechanical model in (Chabiniok et al 2011), and synthetic data with a fully coupled FSI model in (Bertoglio et al 2012b), to estimate some patient-specific characteristics - hence, enabling an additional degree of model personalization - based on a data assimilation strategy.

In this paper, we further demonstrate this strategy applied with FSI in arteries, with a particular focus on taking advantage of the available image data to estimate modeling parameters pertaining to vessel wall boundary conditions. In (Moireau et al 2011), we presented a framework to characterize the impact of external tissues and organs - in particular the spine for the aorta - on the arterial wall, by a simple viscoelastic term prescribed on the surface boundaries of the arterial wall. When applied to an inlet or outlet wall boundary, this viscoelastic term represents the truncated arterial tree extending from the geometric model. Comparably, when applied along the exterior surface of the arterial wall, the viscoelastic term models tethering to the external organs. This approach was shown to dramatically improve the quality of the simulations, providing accurate deformation patterns of the arterial tree. Of course, this requires a careful calibration of the parameters in this boundary support model. To that effect, time-resolved medical image data provides information on the apparent motion of the walls, which is valuable to determine how strong the external tissue support should be.

Whereas the calibration of the boundary support parameters was performed manually in (Moireau et al 2011), in the present article we propose a method to automate and refine this calibration process using the segmentations extracted from the image sequence, without compromising the simulation time which can already be quite long for this type of complex simulation. To accomplish this, we rely on an original estimation frame- work developed in (Moireau et al 2008, 2009; Moireau and Chapelle 2011b) which proposes a strategy for using the available additional time-resolved data to identify various uncertain modeling quantities, while improving the quality of the numerical computations. The possibility to benefit from these additional data in the computation can be contemplated in the framework of data assimilation (Blum et al 2009; Navon 2009), which consists in merging model and data information in order to circumvent the initial model uncertainties and to improve the numerical prediction. In this context, two types of strategies are often considered:

- A variational approach, which estimates the uncertainties by minimizing a least square criterion involving the discrepancy between the data and the corresponding outputs from a model simulation see (Navon 2009; Chavent 2010) and references therein, and (Perego et al 2011) in the context of hemodynamics;

- A sequential approach, which filters the uncertainties over time to stabilize the computed numerical system onto the actual partially-observed system see (Simon 2006) and references therein.

These two approaches are very different in their practical use, but rely on the same fundamental observability condition, which determines that observing the system even partially - through a time sequence of boundary shapes in our study - is sufficient for circumventing the lack of initial knowledge of the system (Simon 2006). Furthermore, in various cases it can be proven that the two strategies are actually equivalent (Kailath et al 2000). With the objective of a fast model identification "on the fly", we adopt herein a sequential approach whereby the uncertainties are evaluated during the simulations by considering the various available observations sequentially. In a very general framework, we consider two classes of uncertainties:

- Parameter uncertainties. These uncertainties are considered by assuming that some of the model constants, in some regions, remain imperfectly assessed after calibration. In particular, in this article we consider that the external tissue support boundary conditions - which constitute a rather simplified model of the complex attachment of the aorta on the spine - are difficult to calibrate manually. Since we define only a few regions with homogeneous coefficients, the total number of parameters to be identified is finite and reasonably small. Note that considering parameters with the same discretization level as the PDE field is possible in theory, but would certainly lead to a very sensitive or even ill-posed identification problem. Moreover, the small num- 
ber of parameters to estimate should allow the use of classical optimal methods in a sequential framework inspired from Kalman filtering, as presented in (Moireau et al 2008; Moireau and Chapelle 2011b) and applied to the identification of elastic properties of arterial walls in the fluid-structure interaction context in (Bertoglio et al 2012b).

- State uncertainties. Other sources of uncertainties sometimes neglected - can be categorized in what we call the state uncertainties. This concerns the lack of knowledge on the initial conditions, the approximation errors coming from the discretization procedure, and even any other uncharacterized model error and approximation. As discussed in (Moireau et al 2008), for a mechanical model these uncertainties always exist in practice and affect the solution fields of the PDEs. Therefore, they should be adequately controlled, i.e. filtered jointly with the identification of the parameters through the data assimilation procedure to avoid an unstable estimation behavior. Furthermore, as shown in (Chapelle et al 2012) in the context of second-order hyperbolic equations, even with perfectly known parameters, filtering the state uncertainties with adequate - reasonably noisy - data always improves the numerical approximation of the system.

Concerning sequential approaches, the main difficulty in taking into account these uncertainties lies in the fact that most of these methods are based on optimization principles which make them not easily suited to PDE systems due to the "curse of dimensionality" coined by R.E. Bellman (Bellman 1957). This is the reason why we adopt here instead an observer strategy which consists in adding to the original model a consistent stabilization term based on the measured discrepancy between the computed outputs of the model at a given time and the observation at hand. When the numerical system matches the actual observations, the stabilization term vanishes. Otherwise, the dynamics of the model are corrected to track the actual trajectory. This idea was initially introduced in the control community (Luenberger 1971), but was only recently applied to PDEs as the so-called Luenberger estimation in (Moireau et al 2008, 2009) or as, in a more recent terminology, the nudging assimilation method in (Auroux and Blum 2008). In this article we present an observer strategy based on (Moireau et al 2009), which can be used with the type of measurements available - i.e., the segmented surfaces of the fluid-structure interface - and has already been studied in its linearized form in the context of a solid coupled with a fluid in (Bertoglio et al 2012a).
The outline of this paper is as follows. First, we recall the model considered for the case of interest and describe the available data. Here, we focus on one of the cases studied in (Moireau et al 2011), a subject in whom the aortic wall motion is rather large, hence the fluid-structure interaction model involves an ALE formulation for the fluid with a non-linear large displacement hyperelastic solid model for the arterial wall. Then, we present our sequential estimator and carefully describe the handling of the specific measurements available here, namely, a time sequence of segmented surfaces. Finally we report on the results and discuss the accuracy, robustness and computational effectiveness of our method.

\section{Models}

We consider here a fluid-structure interaction (FSI) model of blood flow compatible with large displacements of the vessel walls. The model - described in detail in (Moireau et al 2011) - consists of incompressible NavierStokes equations coupled with a visco-hyperelastic formulation. The other salient modeling features concern the boundary condition specification, namely,

- For the fluid, the downstream boundary conditions are modeled using a "coupled multi-domain" approach in which a so-called Windkessel lumped parameter model representing the downstream vasculature provides a dynamic relationship between outlet pressure and flow for the FSI solution (VignonClementel et al 2010);

- For the solid, a visco-elastic support model is used to represent the interaction of the vessel outer boundaries with the surrounding tissues, as well as the effect of the truncated arterial trees. A forcing term was used at the inlet boundary of the vessel wall to account for the heart motion; these simple boundary conditions were found to be very effective in producing simulations that compare well with the available clinical data (Moireau et al 2011).

Lastly, another difficulty arising from patient-specific FSI modeling is that the segmented medical image data correspond to deformed configurations of the vessel walls. Hence, a rather complex inverse problem must be solved in order to determine a corresponding stress-free configuration (Gee et al 2010; Moireau et al 2011), which in turn implies that the initial condition in the solid - also taken as the reference configuration - is pre-stressed. 


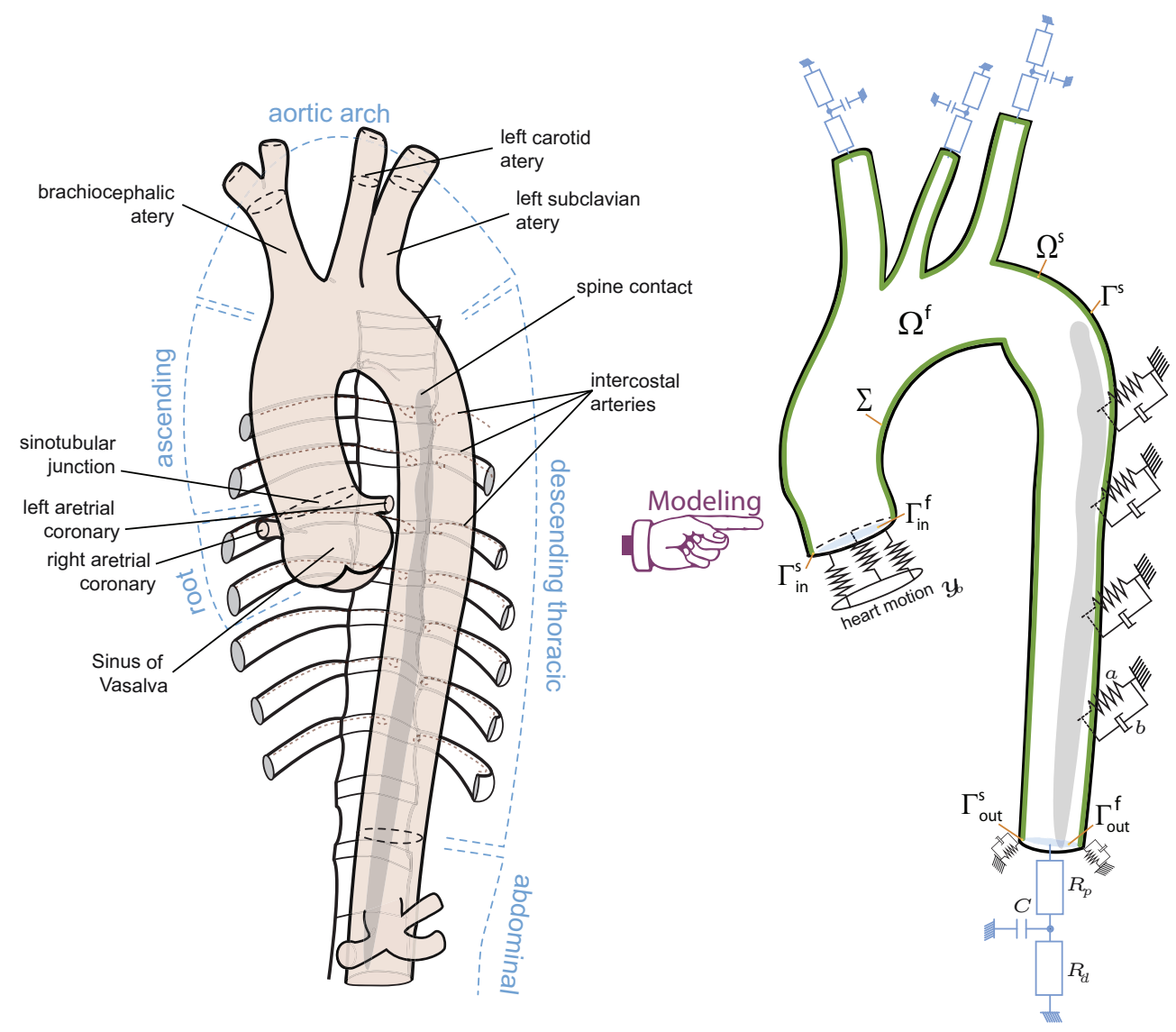

Fig. 1 Schematic representation of aorta and its surrounding organs. Model domain and boundary conditions

\subsection{Model formulation}

We now introduce some notation in order to summarize the model equations. Starting with geometrical and kinematical considerations, we denote by $\Omega^{f}(t)$ the fluid domain at any time $t$, with $\underline{u}_{f}$ the fluid velocity, whereas the solid domain is denoted by $\Omega^{s}(t)$ with $\underline{u}_{s}$ the solid velocity, and $\Sigma(t)=\overline{\Omega^{f}(t)} \cap \overline{\Omega^{s}(t)}$ is the fluid-solid interface, see Figure 1. The deformed solid domain $\Omega^{s}(t)$ is the image of a reference configuration $\Omega_{0}^{s}$ through the solid deformation mapping given by

$\underline{\phi}_{s}: \mid \begin{aligned} \Omega_{0}^{s} \times[0, T] & \longrightarrow \Omega^{s}(t), \\ (\underline{\xi}, t) & \longmapsto \underline{\mathrm{x}}=\underline{\xi}+\underline{y}_{s}(\underline{\xi}, t)\end{aligned}$

where $y_{s}$ denotes the solid displacement, hence $\underline{u}_{s}=$ $\partial_{t} y_{s}$. With a view to an Arbitrary Lagrangian-Eulerian fluid formulation, we also define a fixed fluid domain $\Omega_{0}^{f}$ such that $\overline{\Omega_{0}^{f}} \cap \overline{\Omega_{0}^{s}}=\Sigma_{0}=\underline{\phi}_{s}^{-1}(\Sigma(t))$, and a lifting operator $\operatorname{Ext}^{f}$ (called extension map) from $\Sigma_{0}$ to $\Omega_{0}^{f}$ respecting the fluid Dirichlet boundary conditions on the fluid-structure interface. We can then define the fluid deformation mapping

$\underline{\phi}_{\mathcal{A}}: \mid \begin{aligned} \Omega_{0}^{f} \times[0, T] & \longrightarrow \Omega^{f}(t), \\ (\underline{\xi}, t) & \longmapsto \underline{\mathrm{x}}=\underline{\xi}+\operatorname{Ext}^{f}\left(\underline{y}_{s} \mid \Sigma_{0}\right)\end{aligned}$

which is compatible with the solid mapping in that $\underline{\phi}_{\mathcal{A}}\left|\Sigma_{0}=\underline{\phi}_{s}\right| \Sigma_{0}$. Note that it would be sufficient to ensure the compatibility of the normal components, but it is simpler, and adequate for the present work, to ensure the continuity of the three components. We introduce the corresponding Jacobian $J_{f}(\underline{\xi}, t)=\operatorname{det} \underline{\nabla}_{\underline{\xi}} \underline{\phi}_{\mathcal{A}}(\underline{\xi}, t)$ and the fluid domain velocity $\underline{w}=\partial_{t} \underline{\phi}_{\mathcal{A}}=\operatorname{Ext}\left(\underline{u}_{s} \mid \Sigma_{0}\right)$.

Regarding the remaining physical notation, for the fluid we denote by $\rho_{f}$ the constant mass per unit volume, $p_{f}$ the pressure, $\underline{\underline{\sigma}}_{f}$ the stress tensor given by

$\underline{\underline{\sigma}}_{f}=-p_{f} \underline{\underline{\mathbb{1}}}+2 \mu \underline{\underline{\varepsilon}}\left(\underline{u}_{f}\right)$,

with $\mu$ the dynamic viscosity and $\underline{\underline{\varepsilon}}$ the symmetrized gradient operator. As for the solid we define $\rho_{s}$ as the (constant) mass per unit volume in the reference configuration, $\underline{\underline{\sigma}}_{s}$ the Cauchy stress tensor and $\underline{\underline{\Pi}}$ s the associated first Piola-Kirchhoff stress tensor, both defined based on a hyperelastic potential $W_{s}$, with additional viscous effects represented by a viscous modulus $\eta_{s}$, see 
(Moireau et al 2011) for details. Finally, the strong form of the FSI problem can be written as follows

$$
\left\{\begin{aligned}
\underline{w}=\operatorname{Ext}^{f}\left(\underline{u}_{s} \mid \Sigma_{0}\right), & \text { in } \Omega_{0}^{f} \\
\left.\frac{\rho_{f}}{J_{f}} \frac{\partial J_{f} \underline{u}_{f}}{\partial t}\right|_{\underline{\xi}}+\rho_{f}\left(\underline{u}_{f}-\underline{w}\right) \cdot \underline{\nabla}_{\underline{\underline{x}}} \underline{u}_{f} & \\
-2 \mu \underline{\operatorname{div}}_{\underline{\mathbf{x}}}\left(\underline{\underline{\varepsilon}}\left(\underline{u}_{f}\right)\right)+\underline{\nabla}_{\underline{\mathbf{x}}} p_{f}=0, & \text { in } \Omega^{f}(t) \\
\operatorname{div}_{\underline{\mathbf{x}}} \underline{u}_{f}=0, & \text { in } \Omega^{f}(t)
\end{aligned}\right.
$$

$\left\{\begin{array}{rr}\rho_{s} \frac{\partial \underline{u}_{s}}{\partial t}-\underline{\operatorname{div}}_{\underline{\xi}}\left(\underline{\underline{\Pi}}_{\mathrm{s}}\right)=0, & \text { in } \Omega_{0}^{s} \\ \frac{\partial \underline{y}_{s}}{\partial t}=\underline{u}_{s}, & \text { in } \Omega_{0}^{s} \\ \underline{\underline{F}}^{-1} \underline{\underline{\Pi}}_{\mathrm{s}}=\frac{\partial W_{s}}{\partial \underline{\underline{e}}}+\eta_{s} \underline{\underline{e}}, & \text { in } \Omega_{0}^{s}\end{array}\right.$

$$
\left\{\begin{aligned}
\underline{u}_{f}=\underline{u}_{s}, & \text { on } \Sigma(t) \\
\underline{\underline{\Pi}}_{\mathrm{s}} \cdot \underline{n}_{s}=J_{f} \underline{\underline{\sigma}}_{f}\left(\underline{u}_{f}, p_{f}\right) \cdot\left(\underline{\underline{F}}_{f}\right)^{-T} \cdot \underline{n}_{s}, & \text { on } \Sigma_{0}
\end{aligned}\right.
$$

where $\left.\frac{\partial}{\partial t}\right|_{\xi}$ represents the so-called ALE time derivative, see (Fernández and Gerbeau 2009) and references therein, and $\underline{n}_{s}$ denotes the outward unit normal vector at the boundary of the reference solid domain $\Omega_{0}^{s}$. Note that these modeling equations have been gathered into 3 groups corresponding to the fluid, solid and coupling equations, sequentially. Concerning the solid boundary conditions, we distinguish between $\Gamma_{\text {in }}^{s}$ (see Figure 1), the vessel wall boundary at the inlet face of the model where a time-dependent forcing term $\underline{y}_{b}$ representing the cardiac motion is precribed, and $\bar{\Gamma}^{s}$, the outer vessel wall and outlet face vessel wall boundaries where the viscoelatic tissue support boundary condition is prescribed:

$\underline{\underline{\sigma}}_{s} \cdot \underline{n}_{s}=-k_{s}\left(\underline{y}_{s}-\underline{y}_{b}\right), \quad$ on $\Gamma_{\text {in }}^{s}$,

$\underline{\sigma}_{s} \cdot \underline{n}_{s}=-k_{s} \underline{y}_{s}-c_{s} \underline{u}_{s}, \quad$ on $\Gamma^{s}$.

We do not include a viscous effect on $\Gamma_{\text {in }}^{s}$, primarily to avoid differentiating the displacement $\underline{y}_{b}$ - extracted from the image sequence, hence containing substantial measurement errors - to compute the corresponding forcing velocity. In practice, we adopt a rather large value for the stiffness coefficient $k_{s}$, and thus the elastic boundary condition can be seen as a penalized Dirichlet condition.

As for the boundary conditions for the fluid, the boundary of the fluid domain $\Omega^{f}$ is decomposed into the inlet (connected to the aortic root) $\Gamma_{\text {in }}^{f}$, the outlets $\Gamma_{\text {out }}^{f}$, and the fluid-solid interface $\Sigma$. On $\Gamma_{\text {in }}^{f}$, a velocity field $\underline{u}_{f}=\underline{u}_{f}^{\text {in }}$ is prescribed. The outlet boundary $\Gamma_{\text {out }}^{f}(t)$ is divided into four parts, $\Gamma_{\text {out }}^{f,(i)}, i=1, . ., 4$ denoting the brachiocephalic trunk, left common carotid artery, left subclavian artery and distal part of the thoracic aorta, respectively. On each $\Gamma_{\text {out }}^{f,(i)}$, an outlet pressure $\bar{p}_{i}$ is applied by coupling the Navier-Stokes equations to a three-element Windkessel model. More precisely,

$\bar{p}_{i}=P_{i}^{w}+R_{p, i} Q_{i}$,

where $Q_{i}$ is the volumetric flow rate,

$Q_{i}=\int_{\Gamma_{\text {out }}^{f,(i)}} \underline{u}_{f} \cdot \underline{n}$,

and $P_{i}^{w}$ is the solution to the ordinary differential equation

$C_{i} \frac{d P_{i}^{w}}{d t}+\frac{P_{i}^{w}}{R_{d, i}}=Q_{i}$

The parameters $R_{d, i}, R_{p, i}$ and $C_{i}$ represent the distal resistance, the proximal resistance and the capacitance of the distal vasculature, respectively. The values used in this work can be found in (Moireau et al 2011), Table 1. Lastly, initial conditions must be provided for the velocity and the displacement in the solid, for the velocity and the domain displacement in the fluid, and the four Windkessel pressures $P_{i}^{w}$.

\subsection{Computational aspects}

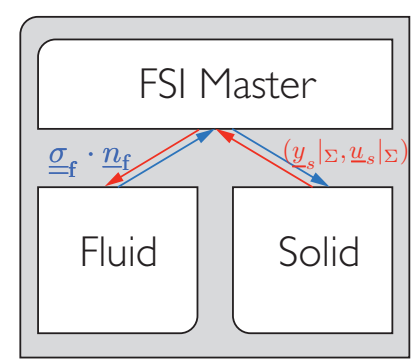

Fig. 2 "FSI-master code" organization where fluid and solid are handled by separate processes which exchange physical quantities at the interface $\Sigma$

In practice we adopt a "multi-code strategy". Concerning the FSI direct simulation in itself, we solve the interaction using a partitioned method inspired from domain decomposition strategies (Fernández and Gerbeau 2009) and a semi-implicit coupling scheme (Fernández et al 2007): at every time step, the advectiondiffusion-ALE stage is solved only once for the sake of efficiency, while the pressure Poisson equation is strongly coupled to the solid, for the sake of stability. This non-linear problem is solved with a Newton algorithm. Besides its sheer effectiveness, this scheme also allows to manage both solid and fluid subsystems in their specific 
implementations with a so-called "FSI-master code" see Figure 2 - that exchanges the Dirichlet or Neuman information requested by each individual code. Therefore, at each iteration of the Newton algorithm the FSImaster:

- receives a fluid "variational residual", corresponding to the load on the interface, and sends it to the solid;

- receives solid displacements and velocities at the interface, and sends them to the fluid.

In our case, the fluid and structure codes were developed independently. The solid model is solved using a classical mid-point time scheme with a $P_{1}$-Lagrange finite element discretization. The fluid system is also discretized using $P_{1}$-Lagrange elements and is stabilized with a streamline diffusion term. The time scheme compatible with the partitioned strategy is a ChorinTeman scheme. On a standard workstation ( 8 cores, 2.5 Ghz) the model solution for a complete heart-beat with a time step of $10^{-3} s$ meshes of about 40,000 vertices - in total for the fluid and the solid - takes about 8 hours, which gives a reference $\mathrm{T}_{\text {ref }}$ timing to which we can compare estimation computational times.

\section{Cardiovascular data}

In this study, we focus on a subject presenting significant thoracic wall motion. The wall motion was acquired in a typical clinical data acquisition setup, as previously presented in (Moireau et al 2011). The data Figure 3(a) - was obtained using a Computed Tomography (CT) angiographic scan - a 64-row Multi-Detector CT scanner (Somatom Sensation Cardiac 64, Siemens Medical Solutions). Ten phases of a 512x512x299 voxel image were reconstructed through the cardiac cycle with temporal resolution of $90 \mathrm{~ms}$, in-plane resolution of $0.62 \times 0.62 \mathrm{~mm}$ and a slice thickness of $1.00 \mathrm{~mm}$. From these images, ten 3D segmentations were generated using a level-set method (Caselles et al 1997) illustrated in Figure 3(b). We then created the corresponding triangular meshes modeling the lumen boundaries (Xiong et al 2010; Moireau et al 2011). These surface meshes of about 6,000 nodes and 12,000 triangles are presented in Figure 3(c).

The computational mesh was obtained from the segmentation corresponding to the diastolic phase after trimming the inlet and outlet to produce flat surfaces suitable for boundary conditions specification in the flow domain - see Figure 3(d). Then, the fluid domain was discretized into a $\mathbb{P}_{1}$-Lagrange tetrahedral mesh. Next, we generated the tetrahedral solid mesh by extruding the lumen boundary triangular mesh with a varying thickness through the model. For example, the thickness of the three smaller arteries - brachiocephalic trunk, left carotid and left subclavian arteries - is set to $1 \mathrm{~mm}$, while we use $2 \mathrm{~mm}$ in the rest of the aorta with a rapid smooth variation at the junctions. The final computational mesh consists of two conforming subdomains, namely, the fluid domain of approximatively 23,000 nodes and 110,000 tetrahedra, and the solid domain of approximatively 18,000 nodes and 24,000 tetrahedra as reproduced in Figure 3(e). We finally divided the exterior boundary of the solid mesh into several regions that have distinct external tissue support characteristics - see Figure 3(e). Each region requires the specification of different numerical values for the coefficients of the visco-elastic boundary conditions. In particular, we identified a "spine region" by locating the area between the origin of the intercostal arteries. Adjacent to this region, we defined a "spine vicinity" region containing the elements of the interface having their nodes within $1 \mathrm{~cm}$ of geodesic distance (Peyré and Cohen 2008) from the spine region. The smaller arteries also define a specific segment for the external tissue support prescriptions, and we further assign separate parameter values on the solid inlet and outlet to set up the artificial truncation boundaries. The rest of the exterior boundary will be considered as a region where the external support is assumed to be light at this stage. This region decomposition will be discussed from a physiological standpoint and possibly refined as described in Figure 7 and in the Discussion section.

As additional patient-specific data, we have recordings of diastolic and systolic pressures. In order to generate the prestress state, we need to associate a stressfree configuration with the solid reference mesh. This is achieved by solving the inverse problem consisting of finding a stress-free configuration that corresponds to a given target deformed geometry under a prescribed loading. In our case the target geometry is the solid reference mesh, and we take the measured diastolic pressure as the prescribed loading.

The above-described sequence of segmented meshes is also used to define the displacements $\underline{y}_{b}$ enforced weakly in our approach - by the heart beats. As described in (Moireau et al 2011), a motion is extracted from the segmentation by identifying the sinotubular junction - separating the sinus of Vasalva from the ascending aorta - and the left coronary artery attachment, see the red circle and red dot in the segmentations of Figure 3(c). In fact, by defining the barycenter of the sinotubular junction, its normal vector and the projection of the left coronary artery attachment point on this section, we can extract a rigid motion. More complex enforced displacement patterns can also be considered by further localizing the right coronary artery attach- 


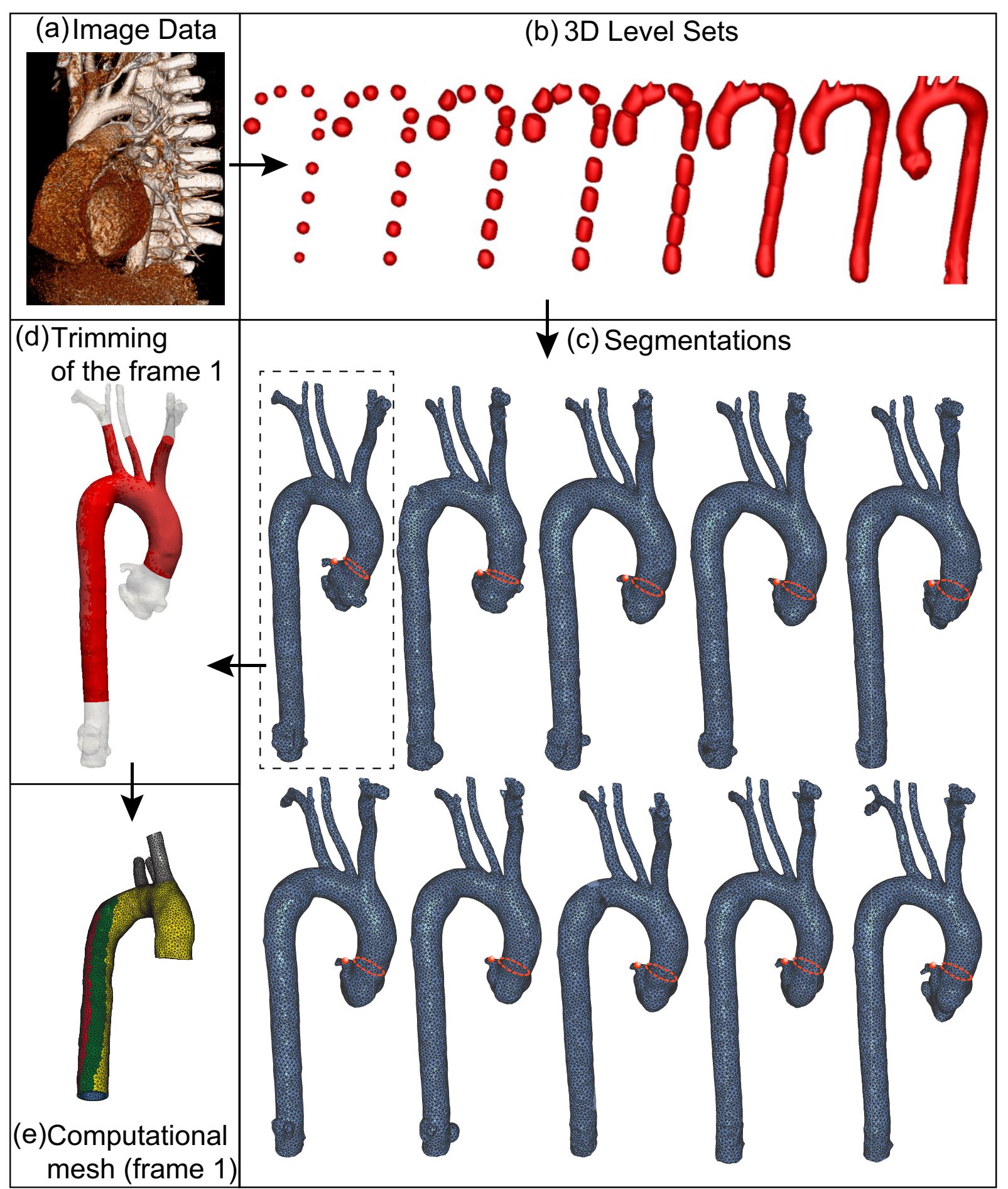

Fig. 3 (a) Volume rendering of image containing one phase of the cardiac-gated CT data. (b) 3D level set is grown to detect the lumen boundaries. (c) Segmentations of the ten phases. The red-circle represents the sinotubular junction and the red dot the left coronary artery attachment. (d) Triming and smoothing of the first frame in order to generate the computational mesh (e) 
ment, but we found that it was not segmented with sufficient accuracy in our ten phases.

The last type of data used in this framework consists of the inlet flows. From physiological considerations on the subject considered, we used an idealized flow waveform mapped to a flat velocity profile to obtain $\underline{u}_{f}^{\text {in }}$ on the aortic root section $\Gamma_{\mathrm{in}}^{f}$. The time variation of the flow is representative of a typical aortic root flow, with a systolic phase spanning over one third of the cardiac cycle and no flow during diastole. This generated a typical cardiac output of 5 liters/minute and a heart rate of 67 beats per minute (Moireau et al 2011).

\section{Sequential estimator}

In this section, we describe our estimation approach by starting with the estimation of the state - meaning the system trajectory - then we extend the strategy to parameter identification. At the end of this section, our methodology is validated with a simple example created with synthetic data.

A sequential estimator - or observer - is a system that recursively uses the data throughout the simulation to retrieve the system trajectory. In the next section, we will see that this idea extends to parameter identification by allowing the estimated parameters to evolve during the simulation to pursue a final value starting from given a priori values. We commonly use a specific notation to distinguish the estimator system from the original system (1). Hence, if we denote by $x(t)=\left(y_{s}(t), \underline{u}_{s}(t), \underline{u}_{f}(t), p(t)\right)$ the system state variables - and in the next section also the additional variable $\theta$ representing the parameters to be identified we now simulate $\left.\underline{\hat{y}}_{\mathrm{s}}(t), \underline{\hat{u}}_{\mathrm{s}}(t), \underline{\hat{u}}_{\mathrm{f}}(t), \hat{p}(t)\right)$ and an additional $\hat{\theta}$ following a modified version of the dynamics (1). The modification - classically called correction - uses the discrepancy between the computed system and the available observation in order to counteract the various sources of uncertainties.

\subsection{State observer}

\subsubsection{Discrepancy measure and state correction}

To introduce this observer, let us first specify how we can compare model and data through a discrepancy measure involving a computed state $x=\left(\underline{y}_{s}, \underline{u}_{s}, \underline{u}_{f}, p\right)$ and a given observation. In fact, assuming sufficient regularity for the real solution and its resulting observation, we can compute at a time $t_{k}$ a discrepancy oper- ator as the signed distance field

$$
\begin{aligned}
& \text { dist }_{\mathcal{S}_{k}}: \\
& \qquad \begin{aligned}
\left(L^{2}(\Sigma)\right)^{3} & \mapsto\left(L^{2}(\Sigma)\right)^{3} \\
\underline{\mathrm{x}}(\underline{\xi}) & \rightarrow \underline{\operatorname{dist}}_{\mathcal{S}_{k}}(\underline{\mathrm{x}}(\underline{\xi}))=\operatorname{dist}_{\mathcal{S}_{k}}(\underline{\mathrm{x}}(\underline{\xi})) \underline{n}_{\mathcal{S}_{k}}(\underline{\mathrm{x}}(\underline{\xi}))
\end{aligned}
\end{aligned}
$$

between the simulated system and the available observed surface $\mathcal{S}_{k}$ with $\underline{n}_{\mathcal{S}_{k}}(\underline{\mathrm{x}}(\underline{\xi}))$ the vector normal to $\mathcal{S}_{k}$ at the projection point. Note that the signed distance allows to determine on which side of the surface the model lies. At every other time, we can then interpolate these signed distance fields to compute appropriate distance fields consistent with the hypothetical position of the unavailable surface $\mathcal{S}(t)$. For example using a linear interpolation we define

dist :

$$
\mid \begin{aligned}
& L^{2}(\Sigma) \times[0, T] \mapsto L^{2}(\Sigma) \\
&(\underline{\mathrm{x}}(\underline{\xi}), t) \rightarrow \underline{\operatorname{dist}}(\underline{\mathrm{x}}(\underline{\xi}), t) \\
&=\left(\left(\frac{t_{k+1}-t}{\Delta T}\right) \operatorname{dist}_{\mathcal{S}_{k}}(\underline{\mathrm{x}}(\underline{\xi}))+\left(\frac{t-t_{k}}{\Delta T}\right) \operatorname{dist}_{\mathcal{S}_{k+1}}(\underline{\mathrm{x}}(\underline{\xi}))\right) \times \\
&\left(\left(\frac{t_{k+1}-t}{\Delta T}\right) \underline{n}_{\mathcal{S}_{k}}(\underline{\mathrm{x}}(\underline{\xi}))+\left(\frac{t-t_{k}}{\Delta T}\right) \underline{n}_{\mathcal{S}_{k+1}}(\underline{\mathrm{x}}(\underline{\xi}))\right) \\
& \text { if } t \in\left[t_{k}, t_{k+1}\right]
\end{aligned}
$$

Based on these distance fields, we can build a discrepancy measure defined for example as the $L^{2}(\Sigma)$ norm of the distance on the surface

$$
\begin{aligned}
D\left(\underline{y}_{s}, t\right) & =\operatorname{meas}_{L^{2}(\Sigma)}\left\{\mathcal{S}_{k}\right\}(x, t) \\
& =\int_{\Sigma}\left|\underline{\operatorname{dist}}\left(\underline{\xi}+\underline{y}_{s}, t\right)\right|^{2} d \Gamma,
\end{aligned}
$$

which is null for the pursued actual displacement field, up to measurement errors and a consistency term deriving from the linear interpolation between two consecutive surfaces, i.e.

$\epsilon_{\Delta T}(t)=D\left(\underline{y}_{s}^{\text {ref }}, t\right)=O(\Delta T)+$ noise.

Therefore, if this discrepancy vanishes - up to the error $\epsilon_{\Delta T}$ - then the given state is consistent with the observations. Note that it does not imply that the simulated system exactly represents the true state, but it indicates that the observation cannot provide any new quantitative information on the quality of our numerical approximation with respect to reality. By contrast, when " $D \neq 0$ ", the simulated trajectory should be corrected with a term that nudges the simulated system in the direction of the actual trajectory. This nudging direction is obtained by differentiating the measured distance with respect to the state. Hence, if we define the interpolated normal vector as

$$
\begin{aligned}
\underline{n}_{\left\{\mathcal{S}_{k}\right\}}(\underline{\xi} & \left.+\underline{y}_{s}, t\right) \\
& =\left(\left(\frac{t_{k+1}-t}{\Delta T}\right) \underline{n}_{\mathcal{S}_{k}}(\underline{\mathrm{x}}(\underline{\xi}))+\left(\frac{t-t_{k}}{\Delta T}\right) \underline{n}_{\mathcal{S}_{k+1}}(\underline{\mathrm{x}}(\underline{\xi}))\right),
\end{aligned}
$$


which is directly related to be the tangent field of the distance - see (Moireau et al 2009) - we can define a discrepancy operator in a variational form on the space of admissible displacements $\mathcal{V}_{s}$ by considering

$$
\begin{aligned}
\mathcal{P}_{L^{2}(\Sigma)} & \left\{\mathcal{S}_{k}\right\}(x, t)\left(\underline{v}_{s}\right) \\
& =\int_{\Sigma} \operatorname{dist}\left(\underline{\xi}+\underline{y}_{s}, t\right) \underline{n}_{\left\{\mathcal{S}_{k}\right\}}\left(\underline{\xi}+\underline{y}_{s}, t\right) \cdot \underline{v}_{s} d \Gamma,
\end{aligned}
$$

for all $\underline{v}_{s} \in \mathcal{V}_{s}$.

The distance (4) considers an $L^{2}$-norm on the interface $\Sigma$ but other norms are possible. In particular, from (Moireau et al 2009; Bertoglio et al 2012a) we have seen that an $H_{\frac{1}{2}}$-norm defined with a lifting operator $\mathrm{Ext}^{s}$ on the solid domain is a more effective discrepancy measure for the state observer. We then introduce

$$
\begin{aligned}
\operatorname{meas}_{H^{\frac{1}{2}}(\Sigma)}\left\{\mathcal{S}_{k}\right\}(x, t) \\
=\int_{\Omega_{0}^{s}}\left\|\operatorname{Ext}^{s}\left(\underline{\operatorname{dist}}\left(\underline{\xi}+\underline{y}_{s}, t\right)\right)\right\|_{\mathcal{E}_{l}^{s}}^{2} d \Omega,
\end{aligned}
$$

with $\|\cdot\|_{\mathcal{E}_{l}^{s}}^{2}$ the norm associated with the linearized elastic energy in the solid, namely equivalent to the $H^{1}$ norm. Therefore its corresponding discrepancy operator operator becomes

$$
\begin{aligned}
& \mathcal{P}_{H^{\frac{1}{2}}(\Sigma)}\left\{\mathcal{S}_{k}\right\}(x, t)\left(\underline{v}_{s}\right)= \\
& \int_{\Omega_{0}^{s}}\left\langle\operatorname{Ext}^{s}\left(\underline{\operatorname{dist}}\left(\underline{\xi}+\underline{y}_{s}, t\right)\right), \operatorname{Ext}^{s}\left(\left(\underline{v}_{s} \cdot \underline{n}_{\left\{\mathcal{S}_{k}\right\}}\right) \underline{n}_{\left\{\mathcal{S}_{k}\right\}}\right)\right\rangle_{\mathcal{E}_{l}^{s}} d \Omega,
\end{aligned}
$$

for all $\underline{v}_{s} \in \mathcal{V}_{s}$. This operator can be shown to be equivalent to

$$
\begin{aligned}
\mathcal{P}_{H^{\frac{1}{2}}(\Sigma)}\left\{\mathcal{S}_{k}\right\}(x, t)\left(\underline{v}_{s}\right)= \\
\int_{\Omega_{0}^{s}}\left\langle\operatorname{Ext}^{s}\left(\underline{\operatorname{dist}}\left(\underline{\xi}+\underline{y}_{s}, t\right)\right), \underline{v}_{s}\right\rangle_{\mathcal{E}_{l}^{s}} d \Omega,
\end{aligned}
$$

using the extension characterization

$\forall \underline{v}_{s}$ such that $\left.\underline{v}_{s}\right|_{\Sigma}=0$,

$$
\int_{\Omega_{0}^{s}}\left\langle\operatorname{Ext}^{s}\left(\underline{\operatorname{dist}}\left(\underline{\xi}+\underline{y}_{s}, t\right)\right), \underline{v}_{s}\right\rangle_{\mathcal{E}_{l}^{s}} d \Omega=0 .
$$

The discrepancy is used as a correction term in the equation relating the solid displacement time derivative to the solid velocity to ensure the stabilization of the state estimator on the actual trajectory as fully analyzed - with linearization arguments - in (Moireau et al 2009). This gives in a weak formulation

$$
\begin{aligned}
\forall \underline{v}_{s} \in \mathcal{V}_{s}, \int_{\Omega_{0}^{s}}\left\langle\frac{\partial \underline{\hat{y}}}{\partial t}, \underline{v}_{s}\right\rangle_{\mathcal{E}_{l}^{s}} d \Omega=\int_{\Omega_{0}^{s}}\left\langle\underline{\hat{u}}_{s}, \underline{v}_{s}\right\rangle_{\mathcal{E}_{l}^{s}} d \Omega \\
+\gamma \int_{\Omega_{0}^{s}}\left\langle\operatorname{Ext}^{s}\left(\underline{\operatorname{dist}}\left(\underline{\xi}+\underline{\hat{y}}_{s}, t\right)\right), \underline{v}_{s}\right\rangle_{\mathcal{E}_{l}^{s}} d \Omega,
\end{aligned}
$$

where $\gamma$ represents a gain parameter to adjust the nudging. Hence, for the complete system (1) in strong formulation we substitute $\left(\underline{\hat{y}}_{s}, \underline{\hat{u}}_{s}, \underline{\hat{u}}_{f}, \hat{p}\right)$ for $\left(\underline{y}_{s}, \underline{u}_{s}, \underline{u}_{f}, p\right)$ in the solid formulation (1b) with the additional identity

$\frac{\partial \underline{\hat{y}}_{s}}{\partial t}=\underline{\hat{u}}_{s}+\gamma \operatorname{Ext}^{s}\left(\underline{\operatorname{dist}}\left(\underline{\xi}+\underline{\hat{y}}_{s}, t\right)\right)$,

replacing the canonical identity $\frac{\partial \underline{y}_{s}}{\partial t}=\underline{u}_{s}$.

The constant $\gamma$ must be carefully calibrated since it represents a trade-off between enhanced stability and the undesirable amplification of the measurement noise, see (Moireau et al 2008, 2009; Bertoglio et al 2012b,a).

\subsubsection{Computational aspects}

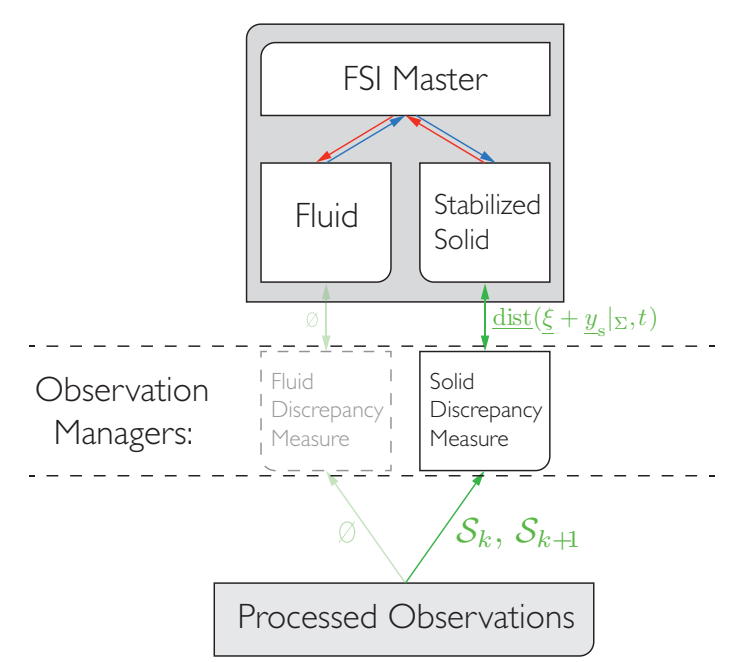

Fig. 4 "FSI-master code" with additional "observation managers" to compute the state observer

Based on the FSI code presented in Figure 2 we handle the computations relative to the observations by interfacing the solid code with an "observation manager" that loads the observed surfaces and computes the discrepancy with a mesh-to-mesh distance algorithm (Baerentzen and Aanaes 2005), as was done in (Moireau et al 2009). When using the state observer model, the modification of the velocity to time-differentiated displacement identity into (8) induces an additional computational cost because velocities cannot be directly eliminated any longer, which doubles the size of the system, hence roughly multiplies the inversion time in the solid by a factor 4 , as explained in detail in (Moireau et al 2009). Since the solid is not the most CPU-intensive part in the complete FSI model this finally only roughly doubles the solution time of the complete problem, meaning about $2 \mathrm{~T}_{\text {ref. }}$. Note that this modified architecture 
presented in Figure 4 can also be supplemented by an analogous "observation manager" on the fluid side if, for example, time resolved velocity or pressure profiles are available - see the end of the discussion for more details.

\subsection{Parameter estimation}

We present here a sequential identification strategy originally proposed in (Moireau and Chapelle 2011b,a), itself inspired from (Pham et al 1998; Pham 2001) and already evaluated in a fluid-structure context in (Bertoglio et al 2012b) for wall stiffness identification where the reader can find the additional specific technical material.

In this work, we concentrate on the estimation of the elastic tissue support $k_{\mathrm{s}}(\underline{\xi})$ representative of the regionalized external tissue tethering. We recall that the available observations are the lumen boundary i.e. the internal boundary of the wall corresponding to the fluid-structure interface $\Sigma$ - whereas we want to identify parameters defined on the external boundary of the walls $\Gamma^{s}$. We must therefore assume that we are confident in the value of the wall stiffness, and we will tackle the question of a joint estimation of external support stiffness and wall stiffness in a future work using additional measurements to differentiate the contributions in the motion of these two types of parameters.

The parameter $k_{\mathrm{s}}(\underline{\xi})$ is a spatialized field and we assume that we can discretize it with a vector $\theta \in \mathbb{R}^{p}$ of small dimension - typically $p<100$ and in this work $p \simeq 10$. Complex spatial representations can be considered in this respect, as done for example in (Xi et al 2011), but we choose here to simply divide $\Gamma^{s}$ into $p$ regions within each of which $k_{\mathrm{s}}(\underline{\xi})$ will be considered as constant. Clearly, the choice of the region division should be consistent physiologically, and we will discuss our estimation results in this light.

\subsubsection{Sequential parameter estimator description}

The identification procedure in a sequential framework consists in adjusting the parameters during the simulation to decrease the discrepancy measure. In other words, the parameters of interest are no longer constant in the estimator and instead evolve with a dynamics of the type

$\frac{\partial \hat{\theta}}{\partial t}=K \mathcal{P}_{*}\left\{\mathcal{S}_{k}\right\}(x, t)\left(\nabla_{\theta} \underline{y}_{s}\right)$,

where $K$ denotes a filter gain operator, $\mathcal{P}_{*}\left\{\mathcal{S}_{k}\right\}$ a discrepancy operator which remains to be specified with respect to its associated norm which in general can differ from that used in state estimation, and $\nabla_{\theta} \underline{y}_{s}$ quantifies the sensitivity of the displacement field with respect to the parameters (Moireau et al 2008; Moireau and Chapelle 2011b). Then the state dynamics should be accordingly corrected relatively to the original dynamics by using the parameter sensitivity, resulting into

$\frac{\partial \hat{x}}{\partial t}=\frac{\partial x}{\partial t}+\left(\nabla_{\theta} x\right)^{\top} \cdot \frac{\partial \hat{\theta}}{\partial t}$,

where " $\frac{\partial x}{\partial t}$ " is an abuse of notation representing the original model equations.

The small dimension of $\theta$ allows us to use optimal filtering to define a filter gain $K$ that is best adapted to a given choice of discrepancy measure. In our case, we choose for simplicity the meas $L^{2}$ definition. The computation of this gain can be approximated by a ReducedOrder Extended Kalman Filtering approach (ROEKF) (Moireau et al 2008) whereby the sensitivity quantities are computed by differentiating the model equations. Alternatively (Moireau and Chapelle 2011b,a) have proposed a Reduced-Order Unscented Kalman Filtering (ROUKF) strategy where, instead of resorting to tangent sensitivity computations, the non-linear impact of the parameters on the state is determined from sampling points representing the parameters uncertainties around the current estimate. To that purpose, we rely on an estimation of the parameter covariance which starting from an initial a priori covariance - will evolve in time, fed by the inverse of the observability Grammian - also called here identifiability Grammian- of the problem (Simon 2006; Moireau and Chapelle 2011b). In this case, we manipulate what we refer to as "uncertainty sensitivities" which map uncertainty directions in the parameter space to associated directions in the state space.

In order to fully summarize the ROUKF approach, it is convenient to write the final equations after space and time discretization of the model. Let us denote by $X^{n}$ the spatial discretization of the state at time $t_{n}$. After discretization of the complete fluid-structure interaction problem we can introduce a forward operator $A^{n}$ which computes $X^{n+1}$ starting from $X^{n}$, i.e.

$X^{n+1}=A^{n}\left(X^{n}, \theta\right)$.

By considering the additional state correction we modify $A^{n}$ into a new operator $A_{\gamma}^{n}$ such that the state observer follows the discrete dynamics

$\hat{X}^{n+1}=A_{\gamma}^{n}\left(\hat{X}^{n}, \theta,\left\{\mathcal{S}_{k}\right\}\right)$.

The purpose of the state estimator is to stabilize the simulated trajectory on the actual trajectory. Of course, 
this is partly artificial as long as the parameters themselves have not been adequately identified, since the parameter error then induces a persistent error source in the model equations. Nevertheless, for parameter identification purposes, remaining in the vicinity of the true trajectory is essential in order for parameter sensitivities to be representative. Then, the parameter identification problem is more likely to be well-posed - and we can prove this in a linearized configuration (Moireau et al 2008). This motivates replacing the classical fluidstructure model $A^{n}$ by the observer $A_{\gamma}^{n}$. Note that in $A_{\gamma}^{n}$ the distance field to the observation surfaces is discretized into a quantity denoted by $D\left(\hat{X}^{n}, t^{n}\right)$. Discretizing the $L^{2}(\Sigma)$-norm with a finite element matrix $M_{\Sigma}$ we have for example

$\mathcal{P}_{L^{2}(\Sigma)}\left\{\mathcal{S}_{k}\right\}\left(\hat{x}^{n}, t^{n}\right)\left(\underline{v}_{s}\right)=V_{s}^{\top}{\frac{\partial D^{\top}}{\partial X}}^{\top} M_{\Sigma} D\left(\hat{X}^{n}, t^{n}\right)$,

for all test functions $\underline{v}_{s} \in \mathcal{V}_{s}$ and $V_{s}$ the vector of the corresponding degrees of freedom. The same logic applies to compute $\mathcal{P}_{H^{\frac{1}{2}}(\Sigma)}\left\{\mathcal{S}_{k}\right\}(x, t)\left(\underline{v}_{s}\right)$, and we refer to (Bertoglio et al 2012a) for technical details.

The ROUKF modifies this dynamics by allowing $\theta$ to evolve in time, and we therefore write $\hat{\theta}^{n}$. The algorithm follows a prediction-correction formalism and is based on sampling points for computing the sensitivities. Given adequate sampling rules, we precompute so-called unitary simplex sigma-points (Julier and Uhlmann 2002) $I_{[i]}, 1 \leq i \leq p+1$ as a discretization of the unit ball in $\mathbb{R}^{p}$. We associate with each sigmapoint a corresponding weight $\alpha_{i}=\frac{1}{p+1}$, and collect the weights in a diagonal matrix $D_{\alpha}$. Then, assuming that the we expect a variation of the parameters given by a covariance ellipsoid around a computed value $\hat{\theta}^{n}$, we can sample particles around $\hat{\theta}^{n}$ using $I_{[i]}$ and the standard deviation associated with the covariance ellipsoid. Hence, from computed $\hat{X}^{n^{+}} \in \mathbb{R}^{N}, \hat{\theta}^{n^{+}} \in \mathbb{R}^{p}$ with additional operators $U^{n} \in \mathcal{S} L_{p}(\mathbb{R})$ (symmetric), $L_{\mathrm{x}}^{n} \in \mathcal{M}_{p, N}(\mathbb{R}), L_{\theta}^{n} \in \mathcal{G} L_{p}(\mathbb{R})$ (invertible), we compute in three steps

1. Sampling:

$$
\left\{\begin{array}{l}
C^{n}=\sqrt{\left(U^{n}\right)^{-1}} \\
\hat{X}_{[i]}^{n^{+}}=\hat{X}^{n^{+}}+L_{\mathrm{x}}^{n} \cdot C^{n \top} \cdot I_{[i]}, \quad 1 \leq i \leq p+1 \\
\hat{\theta}_{[i]}^{n^{+}}=\hat{\theta}^{n^{+}}+L_{\theta}^{n} \cdot C^{n \top} \cdot I_{[i]}, \quad 1 \leq i \leq p+1
\end{array}\right.
$$

\section{Prediction:}

$$
\left\{\begin{array}{l}
\hat{X}_{[i]}^{n+1^{-}}=A_{\gamma}^{n}\left(\hat{X}_{[i]}^{n^{+}}, \hat{\theta}_{[i]}^{n^{+}},\left\{S_{k}\right\}\right), \quad 1 \leq i \leq p+1 \\
\hat{X}^{n+1^{-}}=E_{\alpha}\left(\hat{X}_{*}^{n+1^{-}}\right) \\
\hat{\theta}^{n+1^{-}}=\hat{\theta}^{n+1^{+}}
\end{array}\right.
$$

3. Correction:

$$
\left\{\begin{array}{l}
L_{\mathrm{x}}^{n+1}=\left[\hat{X}_{*}^{n+1^{-}}\right] D_{\alpha}\left[I_{*}\right]^{\top} \\
L_{\theta}^{n+1}=\left[\hat{\theta}_{*}^{n+1^{-}}\right] D_{\alpha}\left[I_{*}\right]^{\top} \\
D_{[i]}^{n+1}=D\left(\hat{X}_{[i]}^{n+1^{-}}, t^{n+1}\right) \\
L_{d}^{n+1}=\left[D_{*}^{n+1}\right] D_{\alpha}\left[I_{*}\right]^{\top} \\
U^{n+1}=\mathbb{1}+w^{-1} L_{d}^{n+1 \top} M_{\Sigma} L_{d}^{n+1} \in \mathcal{M}_{p} \\
\hat{\theta}^{n+1^{+}}=\hat{\theta}^{n+1^{-}} \\
\quad-w^{-1} L_{\theta}^{n+1}\left(U^{n+1}\right)^{-1} L_{d}^{n+1}{ }^{\top} M_{\Sigma} E_{\alpha}\left(D_{*}^{n+1}\right) \\
\hat{X}^{n+1^{+}}=\hat{X}^{n+1^{-}} \\
\quad-L_{\mathrm{x}}^{n}\left(L_{\theta}^{n+1}\right)^{-1}\left(\hat{\theta}^{n+1^{+}}-\hat{\theta}^{n^{-}}\right)
\end{array}\right.
$$

where for every set of particles $X_{[i]}, 1 \leq i \leq p+1$, $\left[X_{*}\right] \in \mathcal{M}_{N, p+1}$ denotes the concatenation of the $(p+1)$ column vectors arranged side by side, and $E_{\alpha}\left(X_{*}\right)=$ $\sum_{1 \leq i \leq p+1} \alpha_{i} X_{[i]}$ computes the empirical mean of the $X_{[i]}$. The scalar $w^{-1}$ is a coefficient playing a similar role to $\gamma$ in the state observer to moderate the parameter correction depending on the measurement noise. In other words, we take into account the observation noise amplitude by considering weighted norms in the choice of our discrepancy measure in the parameter identification process, meaning that we consider $\mathcal{P}_{w^{-1} L^{2}}\left\{S_{k}\right\}(x, t)$, where $w^{-1} L^{2}$ as a subscript means that we use the $L^{2}$-norm weighted by a factor $w^{-1}$.

As described before, we see that based on $L_{X}^{n+1}$ and $L_{\theta}^{n+1}$ which are two operators gathering particles information, a sensitivity operator $L_{X}^{n+1}\left(L_{\theta}^{n+1}\right)^{-1}$ is computed to correct the state dynamics after the parameter update. Furthermore, the operator $L_{\theta}^{n} U_{n}^{-1} L_{\theta}^{n \top}$ can be interpreted as a covariance representation of the parameter variation. Therefore we recognize in $L_{\theta}^{n} C^{n \top}$ a standard deviation used for generating the sampling points starting from the unitary sigma points $I_{[i]}$.

\subsubsection{Algorithm aspects}

From a practical standpoint, the algorithm can be described as follows. At each time step $t_{n}$, for a given estimated state, parameters, parameters covariance and uncertainty sensitivities,

1. Sampling:

- Generate $p+1$ parameters $\hat{\theta}_{[i]}^{n^{+}}$sampling the covariance ellipsoid around the current estimated value;

- Infer the corresponding state sampling points $\hat{X}_{[i]}^{n^{+}}$based on the uncertainty sensitivities;

2. Prediction:

- For each sampling point $\left(\hat{X}_{[i]}^{n^{+}}, \hat{\theta}_{[i]}^{n^{+}}\right)$, solve a onetime step model analysis starting from $\hat{X}_{[i]}^{n^{+}}$at 


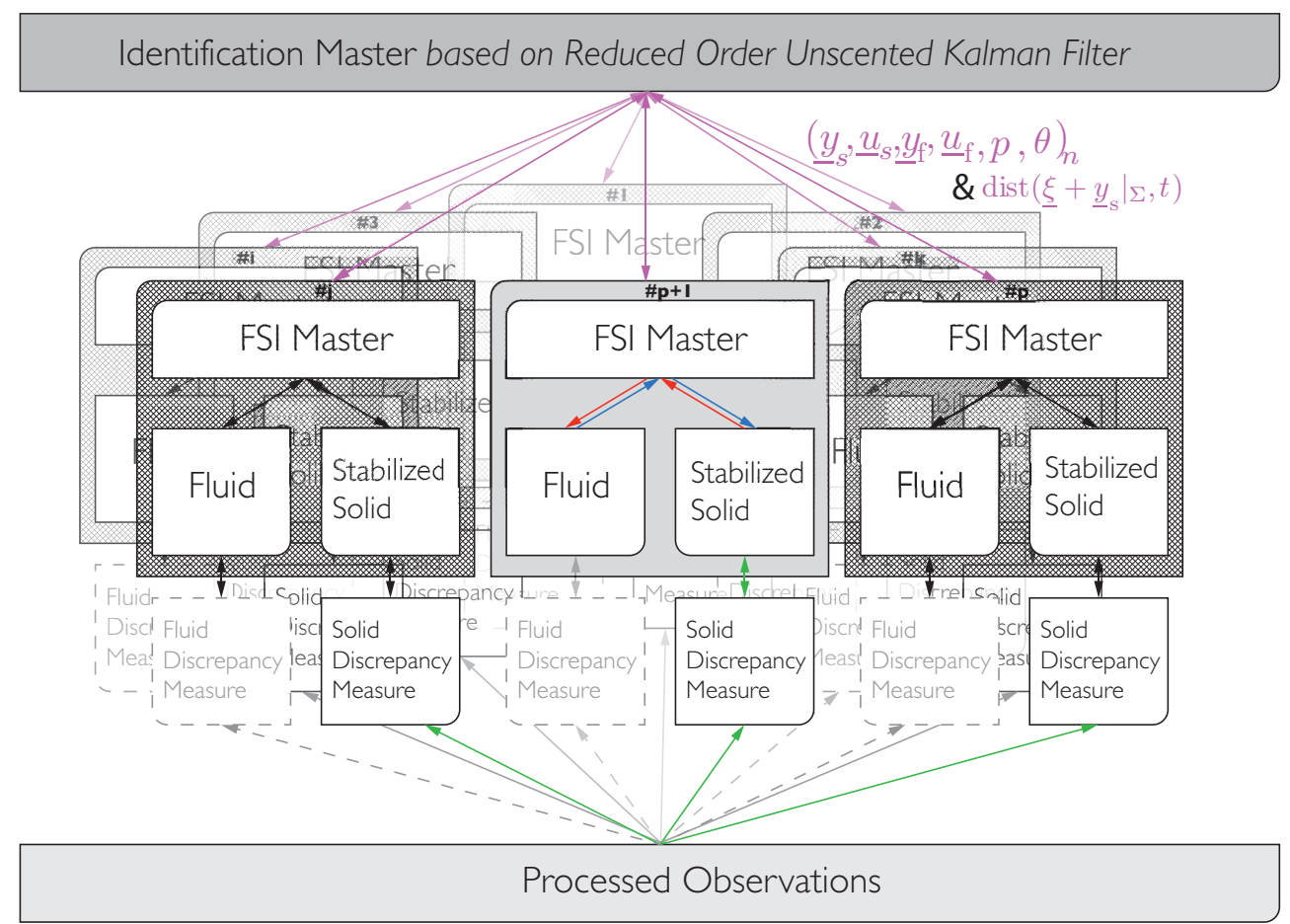

Fig. 5 Complete identification code where the "ROUKF master" receives states, parameters and discrepancies and sends updated states and parameters

$t_{n}$ where the model parameter is set to $\hat{\theta}_{[i]}^{n^{+}}$to produce a predicted state $\hat{X}_{[i]}^{n+1^{-}}$and compute the resulting discrepancy $D\left(\hat{X}_{[i]}^{n+1^{-}}, t_{n+1}\right)$;

- Gather the computed states $\hat{X}_{[i]}^{n+1^{-}}$and infer the new uncertainty sensitivities;

3. Correction:

- Gather the discrepancies. Combined with the uncertainty sensitivities compute the optimal gain;

- Modify the estimated parameters and parameters covariance in consequence;

- Modify the estimated augmented state $\left(\hat{X}^{n+1^{+}}, \hat{\theta}^{n+1^{+}}\right)$by using the sensitivity.

In order to build on the modular architecture of our FSI code, we implement a master code - on top of the FSI-master - in charge of the identification process as illustrated in Figure 5. This "Identification-master" supervises the complete estimation simulation time-step after time-step. It starts by launching in parallel as many FSI-master processes as the identification procedure requires - in our case $p+1$ processes to represent the parametric covariance ellipsoid in a space of dimension $p$. Then each FSI-master receives state-parameter sampling points from the Identification-master to be dispatched to the solid and fluid in order to solve a single time-step evolution. The results are gathered by the FSI-master which forwards them to the Identificationmaster for processing. This strategy has a very limited impact on the original FSI code, and each sampling point time-step simulation can be independently run in parallel. Hence, a complete heart beat simulation with parameter identification is only marginally longer than the previous $2 \mathrm{~T}_{\text {ref }}$ with a CPU-overhead almost entirely due to communications.

\subsubsection{Synthetic test case for algorithm evaluation}

Before moving to the actual results section where we will use the clinical data presented in Section 3, we first validate our complex estimation framework with a simplified problem using synthetic data. We consider a straight tube of dimensions comparable to the descending aorta with a $2 \mathrm{~cm}$ radius and $10 \mathrm{~cm}$ length. On this simplified geometry, we define 4 regions. The first one represents the "spine" region surrounded by 2 "vicinity" regions, and the fourth region corresponds to the "opposite" to the spine as represented in Figure 6. We define in each region a specific parameter value for the stiffness coefficient of the external tissue support condition in the form

$k_{\mathrm{s}}(\underline{\xi})=2^{\theta_{i}}$, on $\Omega_{i} \quad 1 \leq i \leq 4$.

The corresponding mesh of about 1,000 nodes and 4,000 tetrahedra for the solid, and 2,000 nodes and 10,000 tetrahedra for the fluid, was designed to be representative of the target system without undue com- 


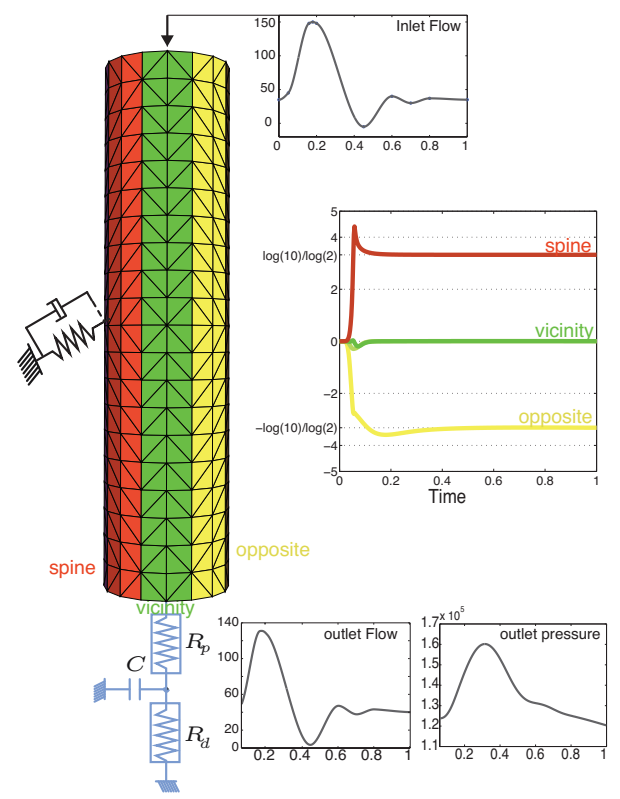

Fig. 6 Synthetic case corresponding to an idealized descending thoracic aorta. Estimation of 4 parameters (spine, vicinity left, vicinity right, opposite) with synthetic noise-free surfaces

putational complexity to efficiently evaluate the identification algorithm. In this regard, the input flow was defined from the flow extracted from the real aortic simulation in (Moireau et al 2011) at the junction between the aortic arch and the descending aorta, and the Windkessel element parameters are also taken from (Moireau et al 2011). By setting $\theta^{\text {ref }}=\left(\frac{\log (10)}{\log (2)}, 0,0,-\frac{\log (10)}{\log (2)}\right)^{\top}$ corresponding to $2^{\theta}=(10,1,1,0.1)^{\top}$ we generate synthetic surfaces at every time step. Then the identification procedure is performed starting from the a priori $\hat{\theta}(0)=\theta_{0}=(0,0,0,0)^{\top}$. The parametric covariance is set to identity and the noise covariance is purposely low since the generated data are noise-free, in order to only evaluate the identification strategy. The parameter evolution during the sequential identification procedure is presented in Figure 6, and we conclude to the very fast convergence of the estimated parameters to their reference values.

\section{Clinical data results}

In this section we will present estimation results obtained with the above-described clinical data when applying our proposed methodology. In order to provide quantitative comparison references we will compute some numerical errors based on distances between contours in several sub-regions of the fluid-solid interface, namely, the lumen contour. These regions are depicted in Figure 7, which also illustrates the comparison and distinction of the previously introduced boundary support regions subdividing the outer wall. Here, we first compare in Figure 8 contours extracted from the image sequence to the initial configuration of the model - obtained after regularization of the extracted contour in the first image - in order to provide an estimate of the apparent motion of each sub-region, both in $L^{2}$ and $L^{\infty}$ norms. This should give valuable comparison references for the next results of this paper

We also reproduce in Figure 9 some error plots already given in (Moireau et al 2011) and providing the distances between the segmented contours and the lumen boundaries of the model simulated with homogeneous Dirichlet conditions prescribed at the inlet and outlet as only essential boundary conditions for the wall, namely, without tissue support. This shows that the errors induced by this naive modeling setup are of the same order as the motion itself, and even much larger in some areas such as the spine and its vicinity.

\subsection{State estimation results}

In this section we illustrate the efficacy of the state estimator in robustly tracking the actual trajectory. In the absence of absolute ground truth, we consider some reference direct simulations produced with parameter values manually calibrated to represent the external tissue support as in (Moireau et al 2011), where these support conditions were shown to significantly improve the distance errors compared to the simplistic boundary conditions considered in Figure 9.

We now compare the standard simulation with boundary support derived from System (1) and the corresponding state estimator - where we modify (1b) using (8) - for an initial condition error that should be adequately tackled by the estimator. To that purpose, we generate the initial condition by running the standard simulation during one cardiac cycle and saving the last configuration. In the results presented in Figure 10, we compare the results obtained with increasing values of the gain $\gamma$ characterizing the nudging effect, in order to obtain a satisfactory trade-off between the dynamics stability improvement and the data noise amplified by the gain. The first row shows the error results obtained with a zero gain, namely, a direct simulation. The second row displays the errors corresponding to a finite gain value $\gamma_{0}=3(\mathrm{cgs})$ and we observe a decrease of the distances in all regions as expected, even if the parameter values are only manually calibrated. The third row corresponds to $\gamma=5 \gamma_{0}$, but convergence in the simulations was only obtained after restricting the observation surfaces in the estimator to disregard an area of $1 \mathrm{~cm}$ extent along the vessel, starting from the inlet ring on which large displacements are prescribed. This 


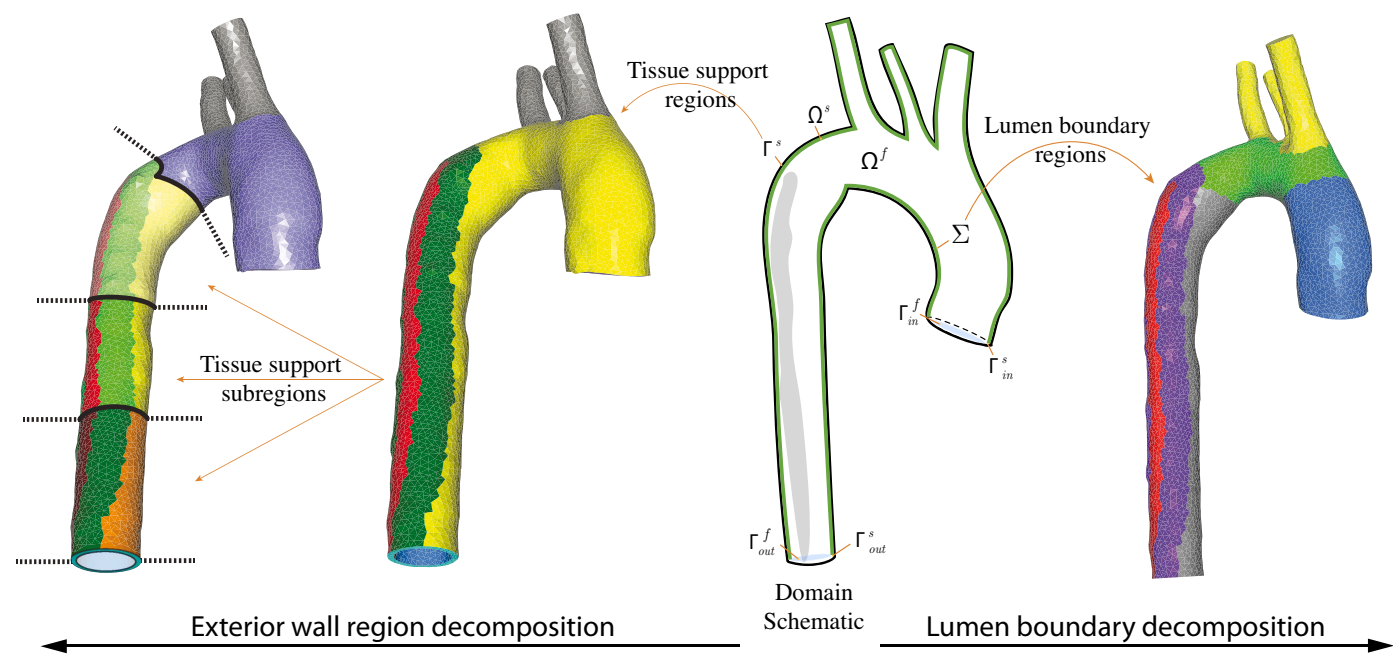

Fig. 7 Anatomical schematic (second right) underlying the hierarchical tissue support division of the outer wall (to the left) and the division of the fluid-structure interface for error computations (to the right)

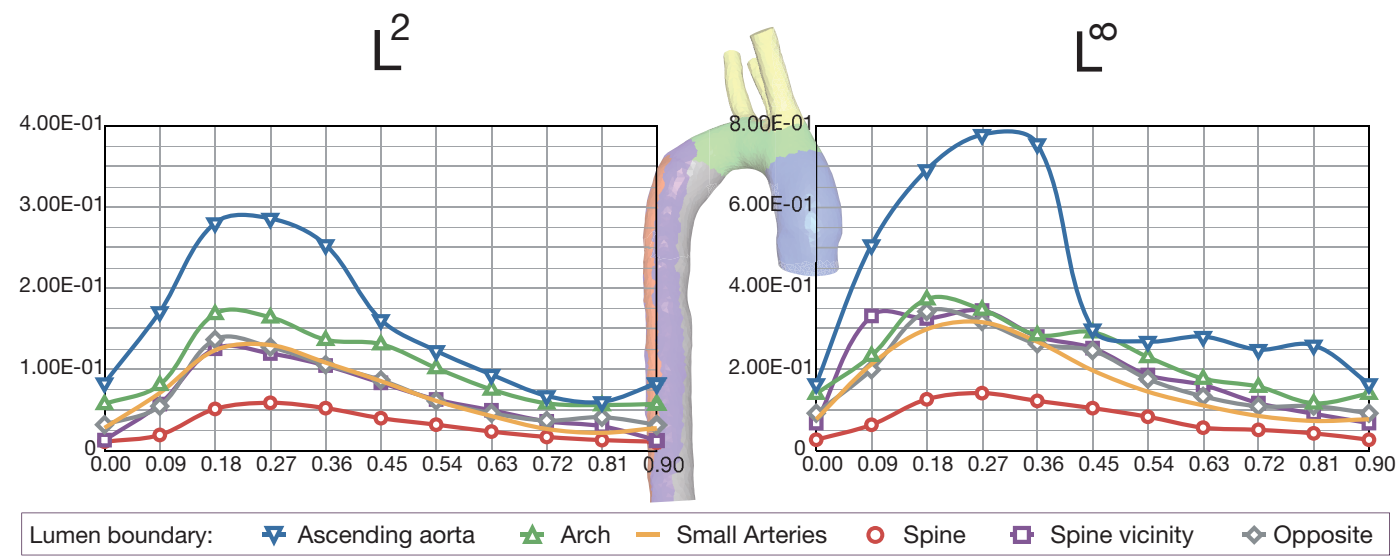

Fig. 8 Apparent motion analysis $-L^{2}$ and $L^{\infty}$ (left and right resp.) norms of distances from the segmented lumen in each image with respect to the initial configuration of the model - Distances versus time plots in cgs units (for comparison, vessel radius is approximately $1 \mathrm{~cm}$ )

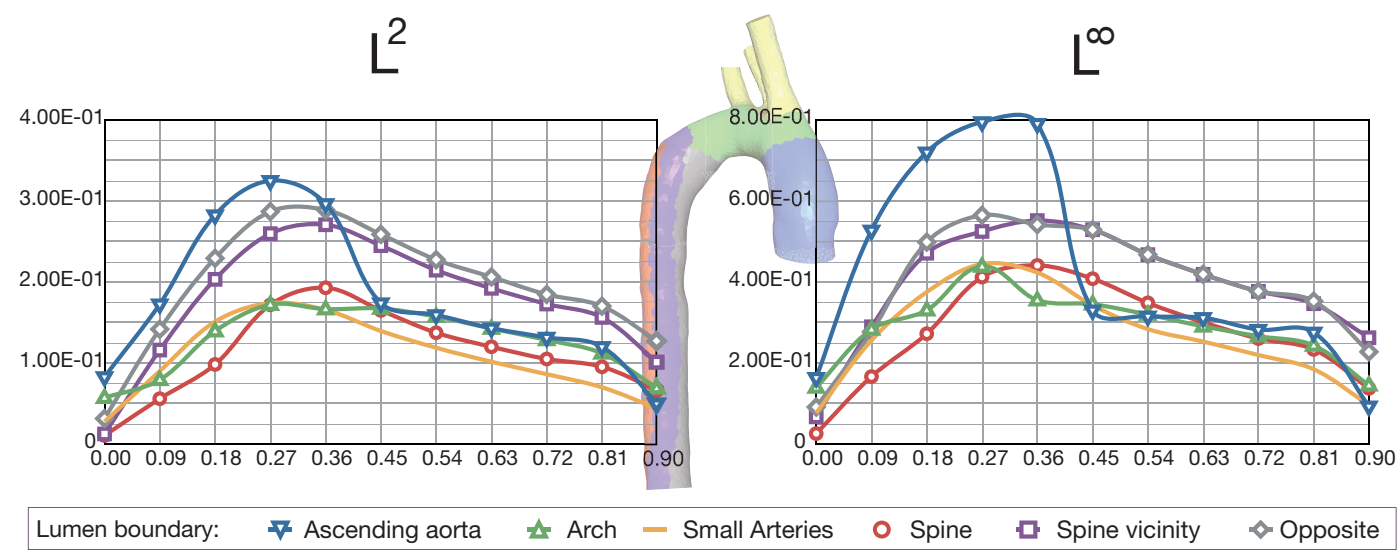

Fig. $9 L^{2}$ and $L^{\infty}$ (left and right resp.) norms of distances from the simulated fluid-structure interface of a model with fixed inlet and outlet as only Dirichlet boundary conditions to the segmented lumen surfaces in the image sequence - Distances versus time plots in cgs units (for comparison, vessel radius is approximately $1 \mathrm{~cm}$ ) 


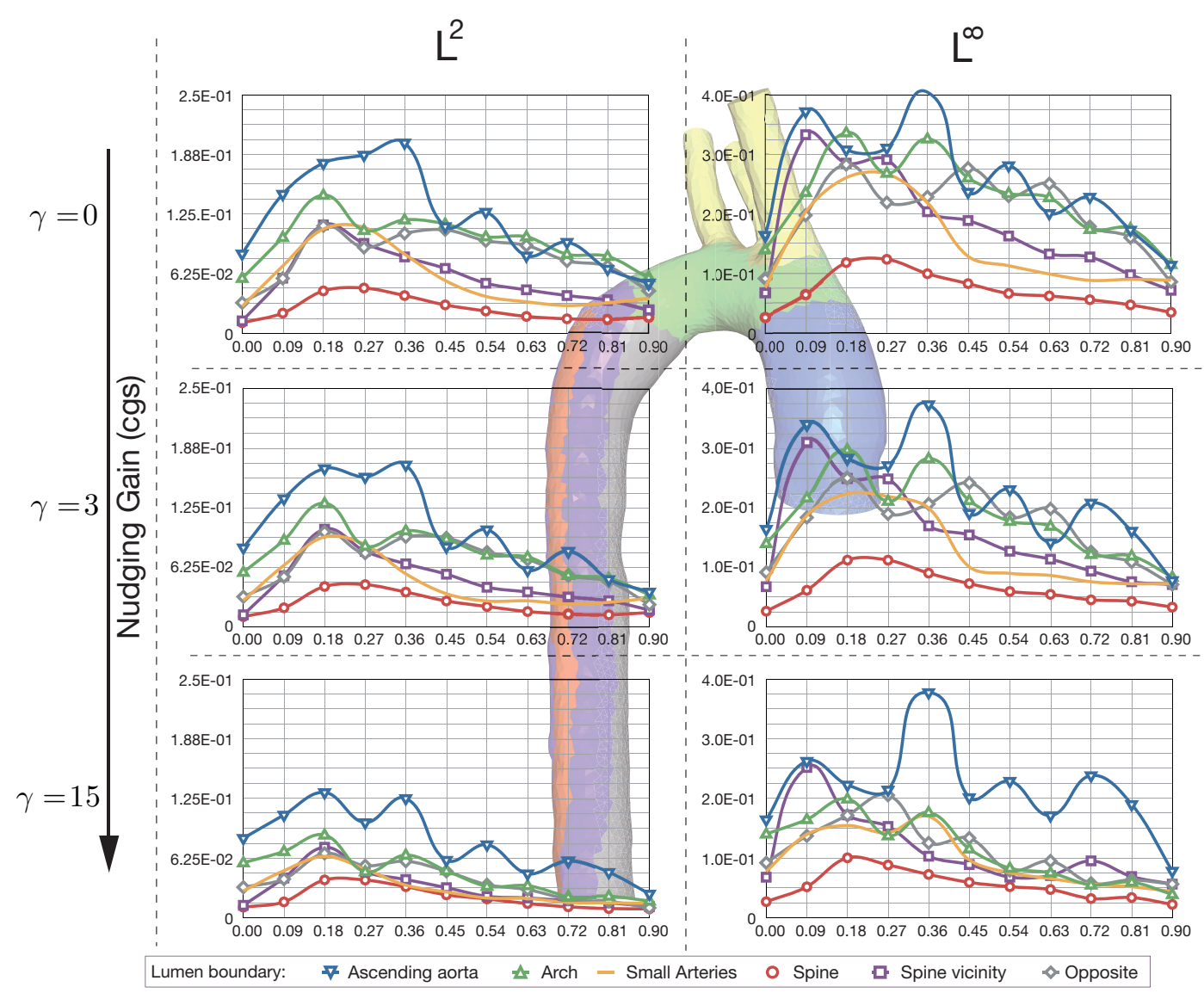

Fig. $10 L^{2}$ and $L^{\infty}$ (left and right resp.) norms of distances from the segmented lumen boundary to the simulated model fluid-structure interface using tissue support with additive state observer and initial condition error - Distances versus time plots in cgs units (for comparison, vessel radius is approximately $1 \mathrm{~cm}$ )

was necessary to avoid difficulties in the ALE mesh motion computation induced by large prescribed displacements directly adjacent to regions where strong estimation corrections are applied. In fact, for $\gamma=10 \gamma_{0}$ such difficulties with the ALE mesh appear with the estimation corrections alone - i.e. away from any boundary condition - which draw the boundaries toward segmented surfaces featuring limited regularity. Note additionally that using a very large gain also reduces the system identifiability, see the Discussion section below. Finally, for all these reasons we select $\gamma=\gamma_{0}$ for all our subsequent estimation computations.

\subsection{Parameter identification}

We now consider a joint state and parameter estimation of 4 regions of interest - see Figure $7,2^{\text {nd }}$ left. The first three regions are the "spine" region, one "spine vicinity" region surrounding the spine, and a "light support" zone aggregating the region opposite to the spine with the arch and the ascending aorta. In these three regions we identify the external tissue support stiffness representing the external tethering. The last region is made of the outlet surface ring at the end of the descending aorta. In this region we also want to estimate the stiffness parameter of the tissue support boundary condition which, here, artificially represents the rest of the arterial tree. We gather the region parameters corresponding to the 4 regions ("spine", "spine vicinity", "light support", "outlet") in a vector $\theta=\left(\theta_{i}\right)_{1 \leq i \leq 4}$, and define as we did for our validation test case

$k_{\mathrm{s}}(\underline{\xi})=2^{\theta_{i}}$, in $\Gamma_{i}^{s} \quad 1 \leq i \leq 4$.

In this real case estimation, we are primarily interested in the external support of the aorta itself, hence we use prescribed (small) values of $k_{\mathrm{s}}(\xi)$ as calibrated in (Moireau et al 2011) for the "small arteries". Furthermore, the artificial inlet support is large enough to assume that the heart motion is almost imposed as a Dirichlet boundary condition and does not necessitate an actual identification. We also refer to Section 6 for further discussions on whether or not to include these quantities in the estimation.

We then consider two starting a priori choices: 


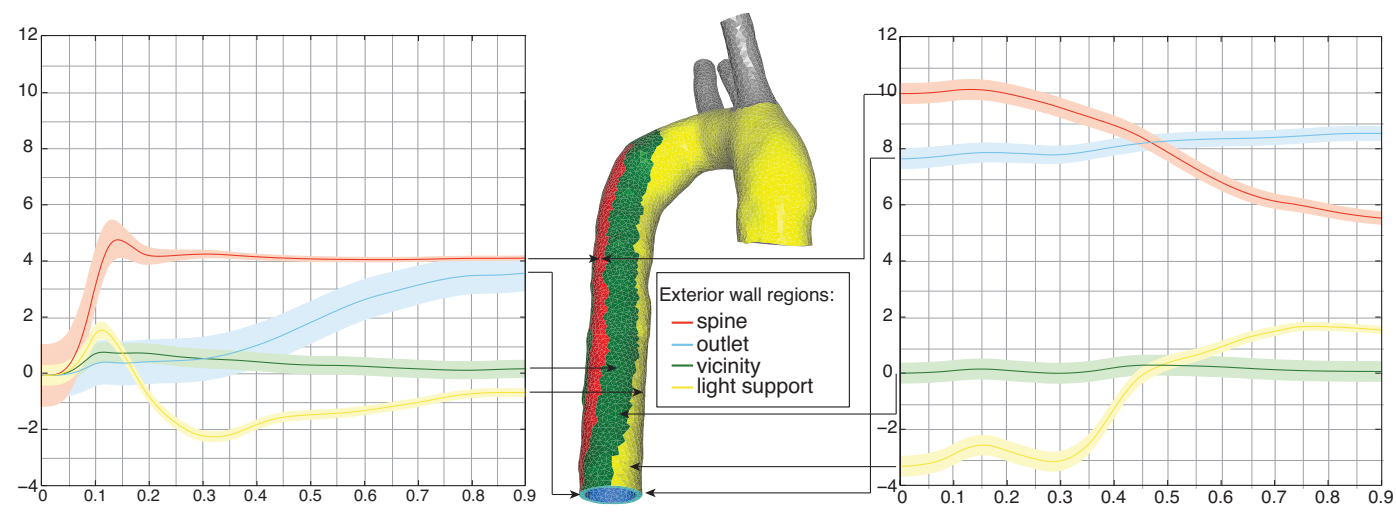

Fig. 11 Time identification of the 4 parameters using real data - Standard deviations diagonal coefficient $\left(L_{\theta}^{n} C^{n} \mathbf{T}\right)_{i, i}$ for $i=1 . .4$ are represented by a colored band around the mean value

1. Firstly, we choose $\theta_{0}=0$ for all regions and the covariance is diagonal with

$$
\begin{aligned}
P_{0}(1,1) & =1.25, \\
P_{0}(i, i) & =0.125, \quad\{i=2,3\}, \\
P_{0}(4,4) & =0.625,
\end{aligned}
$$

which reflects our a priori on the expected variations of the parameters $\theta_{1}$ and $\theta_{4}$ for the "spine" and "outlet", respectively.

2. Secondly, we use $\theta_{0}=\theta^{\text {manual }}$, the value manually calibrated in (Moireau et al 2011) and

$P_{0}=\alpha \mathbb{1}$, with $\alpha=0.125$,

to represent the fact that we assume the parameters should vary around the a priori value with the same expectation.

Considering the observation noise amplitude we choose a weighted $L^{2}$-norm in the choice of our discrepancy measure in the parameter identification process and set $w=10^{-2} / 180$ calibrated to represent the amplitude of the measurement noise covariance (about $1 \mathrm{~mm}^{2}$ in cgs) divided by the surface of the regions measured of about $180 \mathrm{~cm}^{2}$ - to cancel the surface extent effect in the norm. Note that in practice after discretization in time of the model, this term is multiplied by $\frac{\delta t}{\Delta T}$ the ratio of the time discretization $\delta t$ time-step versus the data time sampling $\Delta T$ to take into account the difference of time-sampling between the simulation and the data. Indeed, it is logical to decrease the covariance when the data sampling increases, and when we increase the model accuracy for a given data time-sampling.

The results in Figure 11 show the evolution of the parameters in the course of the sequential estimation process, for the above two choices. The solid line represents the estimated mean value, whereas the corresponding colored bands show the estimated standard deviation around the parameters used by the algorithm to evaluate the sensitivity matrices. The band shrinking around the mean value indicate a strong confidence in the identification. The parameters associated with the "spine" region and the "outlet" converge toward positive and rather large values, while the "spine vicinity" coefficient remains close to 0 indicating a light spine tethering in this region. However, we see oscillations in the parameter evolution of the "light support" region reflecting a lack of convergence in the identification process for this particular parameter. The observations are consistent for two starting a priori choices and we further compare the respective estimation results in Section 6.

In the absence of absolute ground truth, following (Chabiniok et al 2011) we validate these first results with a second estimation procedure using more regions. In particular, the descending aorta is subdivided into 3 segments - see Figure 7 left - leading to 3 "spine" subregions, 3 "spine vicinity" subregions and 3 "opposite to spine" subregions, now distinct from the separate "arch" region. The initial covariance is set proportional to identity - i.e. $P_{0}=\alpha \mathbb{1}$, with $\alpha-0.067$ - since we start from the estimated values obtained with the previous 4 regions. Figure 12 shows the evolution of these 11 parameters with a stable convergence for all values except for the "arch" region which continues to oscillate.

We finally run a direct simulation - namely without the identification procedure - using the estimated values and compare the distances with the results obtained with very light external tissue support in these 4 regions - i.e. $\theta_{0}=0$ - or with the calibrated values of (Moireau et al 2011) - i.e. $\theta=\theta^{\text {manual }}$. The computed distances are presented in Figure 13, and we also display in Figure 14 a comparison of the simulated contours with CT images at peak systole, as well as computed fluid pressures and velocities at the inlet and outlets. 


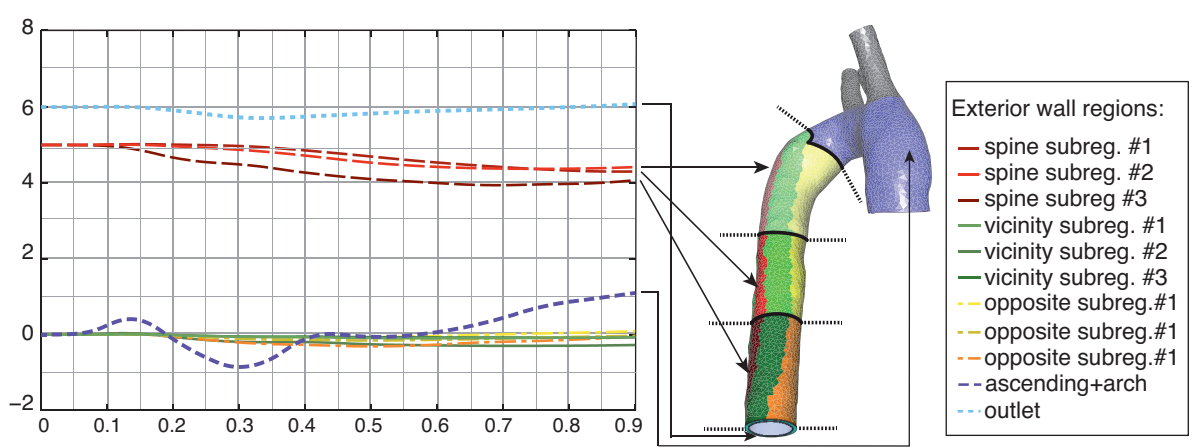

Fig. 12 Time identification of the multi-regions parameters (11 values) using real data

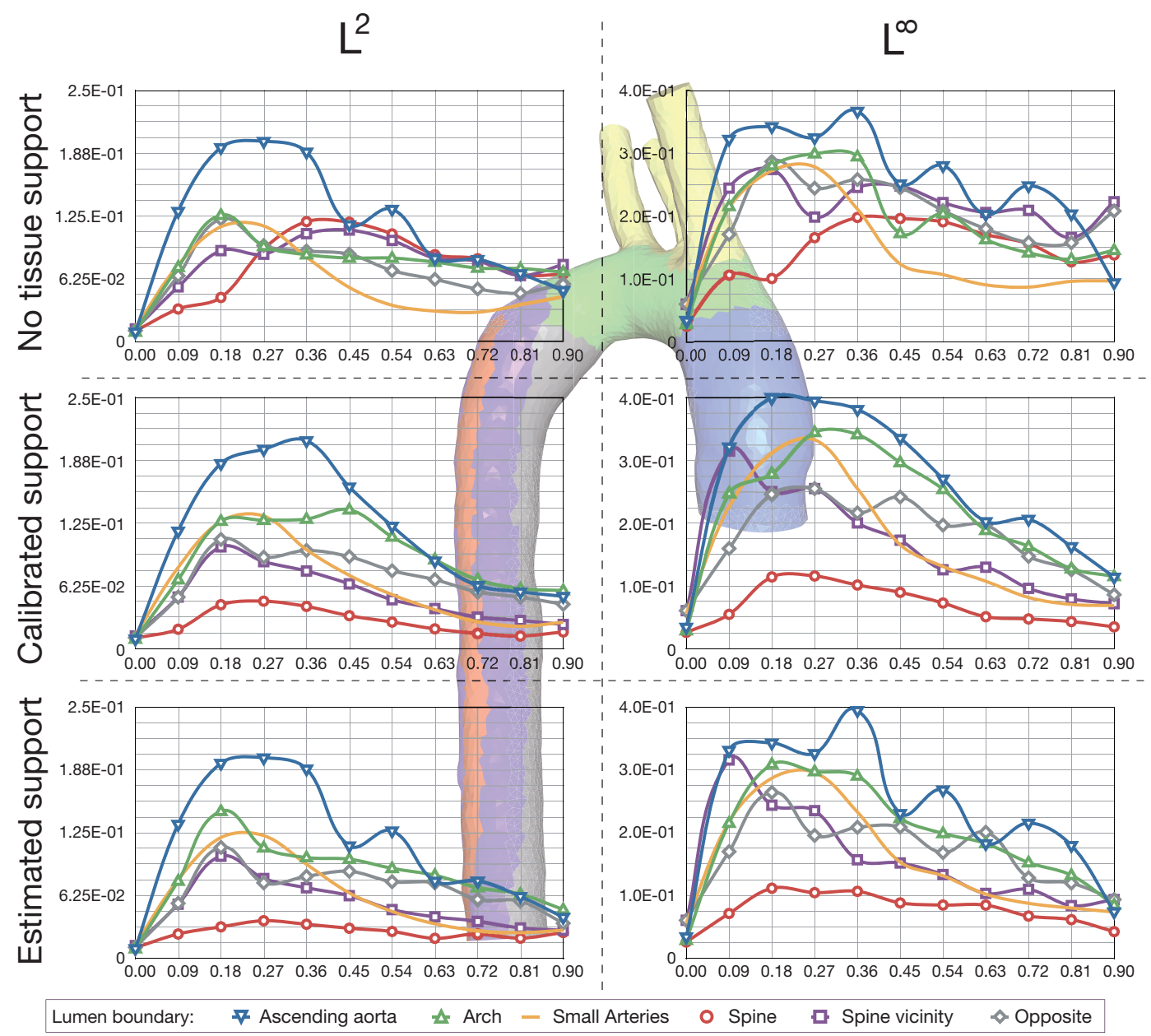

Fig. $13 L^{2}$ and $L^{\infty}$ (left and right resp.) norms of distances from the segmented lumen to the simulated fluid-structure interface using the heart motion only (top) with additive manually calibrated tissue support (middle) and estimated tissue support (bottom)

\section{Discussion}

Successfully identified parameters - The results demonstrate that we obtain stable and consistent identified parameters for the "spine" region and its vicinity, throughout the various estimation setups considered above, namely, with different initial parameter and covariance values (Figure 11), and when increasing the number of regions (Figure 12). Note that the final estimated parameter in the "spine" region is in fact smaller than the calibrated value of (Moireau et al 2011), which is found to be justified when closely examining in Figure 8 the apparent motion extracted from the segmented images. Indeed, the calibrated value in (Moireau et al 2011) leads to almost no motion in the spine region, 


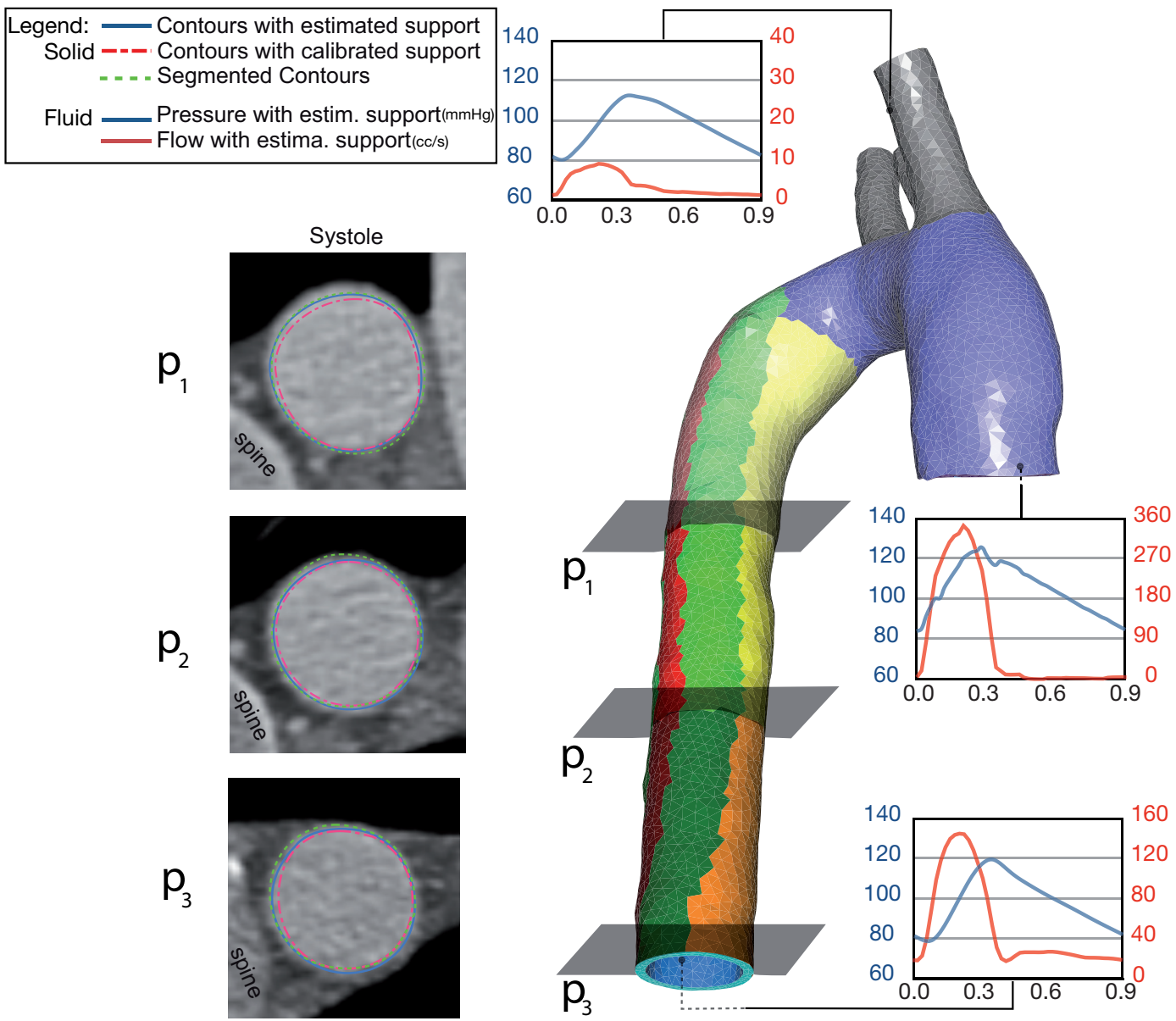

Fig. 14 Left: comparison of segmented (dashed green) vs. simulated (blue with estimated values and pink with manually calibrated values) lumen contours at peak systole - Right: computed fluid pressures and velocities at inlet and outlets

whereas we see in Figure 8 that this part is not completely fixed, see also Figure 14.

A slightly less consistent - although still stable behavior is observed for the outlet parameter. In this case, as we will see below with Figure 15, identifiability is not an issue since this parameter converges very fast to its synthetic value when using synthetic data. However, this parameter corresponds to an ad hoc modeling ingredient compensating the truncation of the arterial tree, hence it certainly does not have an absolute value independently of other modeling parameters, and in particular its adjustment can be expected to depend on the spine support parameter value.

This validates the double interest of our solid boundary terms in order to represent (1) physical boundary conditions accounting for external anatomical structures, and (2) adjustable artificial boundary conditions modeling the rest of the arterial tree. Moreover, this also indicates that no tissue support is really necessary in the "spine vicinity" and "light support" regions.
Identifiability considerations regarding heterogeneous regions - The parameter associated with the region containing the arch shows an oscillating behavior, typical in sequential estimation of non convergence due to identifiability issues. We claim that this is due to two main reasons. First, the computation of distances between two complex curved geometries like arches is not very informative. In fact, for a straight geometry with a main deformation normal to the surface, the distance to the target object is a good estimate of the displacement error between the current simulation and the target system. However, for the aorta, taking into account the large displacements induced by the heart, the distance computation in the aortic arch does not allow to follow actual material points. Then various configurations can lead to a rather small discrepancy measure and the total identifiability is reduced. Secondly and probably more importantly, the region called "light support region" in this paper in fact consists of heterogeneous components which are: the "opposite to the spine" part, the "arch" in itself and even the ascending aorta. Each region cor- 
responds to a different motion and behavior and also to a different tissue support - for example the arch is in reality sustained from below by the pulmonary trunk. Hence, by aggregating heterogeneous regions in a single region we decrease the identifiability of each region in itself.

These remarks are corroborated by our estimation presented in Figure 12 where we subsampled the 4 initial regions into 11 regions. In this estimation starting from the converged parameter values obtained with 4 regions, we see that the estimation is stable for the "spine" and "vicinity" subregions. Furthermore the "opposite to the spine" subregions do not exhibit parameter oscillations anymore and these estimated values indicate the expected light support behavior. However, in the last region corresponding to the heterogeneous aggregation of the arch and the ascending aorta the parameter continues to oscillate.

Data time sampling identifiability issues - The accuracy of the estimation should also be interpreted in the light of the limiting identifiably due to the time sampling of the real data which is quite low, i.e. $\Delta T=$ $10^{2} \delta t=0.1 T$. To illustrate this argument we present in Figure 15 an additional estimation procedure with synthetic data using the real geometry. Here, we generate target surfaces with two different sampling rates: a reasonably high resolution $\Delta T=10 \delta t=0.01 T$, and a low resolution $\Delta T=100 \delta t=0.1 T$ analogous to the real data resolution. The observations correspond to a model where the external tissue support parameters are those manually calibrated in (Moireau et al 2011), $\theta^{\text {ref }}=\theta^{\text {manual }}$. Then the identification is performed with an initial state error as in Section 5.1, and with zero a priori values for all parameters. For these estimations, we consider a rather small observation noise covariance $\left(w=10^{-2} w_{\text {real }}\right.$ with respect to the value chosen in the real configuration) identical for the two sampling rates, arguing that the observation surfaces are noise-free since they are originally synthetically produced by the model. However, there still exist some errors in the observations due to time sampling and the resulting linear interpolation used in the estimation sequence. The impact of this sampling error is therefore purposely amplified by the small observation noise covariance.

We see that the outlet solid boundary condition is well estimated in absolute value already with a low sampling rate, which confirms its good identifiability. Concerning the spine external tissue support we see that the sensitivity is correct with the two sampling rates even if we start from a wrong a priori. However, absolute value convergence is obtained only with the higher sampling rate. Finally concerning the "vicinity" region and the "light support" region, accurate trends can be globally observed since the "vicinity" region parameter value is in between the "spine" region parameter value and the "light support" region parameter value. However absolute values are incorrect. We argue that it is mainly due to an identifiability issue in the "light support" region - as developed above - which contaminates the "vicinity" region. Note that since the noise covariance is low, all effects are magnified in this illustrative example.

Effect of the state estimator - In fact, conceptually the essential purpose of the state estimator is to remain "sufficiently close" to the actual trajectory during the identification process while the parameters evolve in time. We emphasize that this idea is fundamentally distinct from the objective of making the simulated system simply "fit the data", namely driving the computed discrepancy to zero, which is the main purpose of data-based tracking. In the case of filtering estimation, by contrast, the objective is achieved by concurrently nudging the system "towards the data" and enhancing the stability of the simulated system. This induces some specific constraints in the choice of the state estimator gain, in addition to concerns related to measurement noise amplification as already seen in tracking. Namely, in many types of dynamical systems of interest - as e.g. in solid continuum mechanics and FSI - making the gain parameter arbitrarily large has a detrimental impact on the stability (Moireau et al 2008, 2009). Moreover, as already discussed and assessed in (Chabiniok et al 2011), using a very large gain also reduces the system identifiability, since the state estimator would then follow the observation regardless of the parameter values, which would compromise the concurrent parameter identification process.

Integration of multiple observations and material parameters - In the work presented here, the observations were given by a set of medical image data segmentations defining the position of the fluid-structure interface $\Sigma$ through the cardiac cycle. This observation is therefore concerned with a single state variable: the (Eulerian) motion of the fluid-solid interface. However, in a more general setting, one may have to deal with multiple observations of different state variables, such as recordings of pressure and flow waveforms, in addition to the aforementioned motion of the fluid-solid boundary. These different observations of the state must therefore be considered simultaneously in the state and parameter estimation endeavor. Pressure can be acquired noninvasively via a sphygmomanometer, or invasively via a cannula needle placed through an arterial line. Flow 

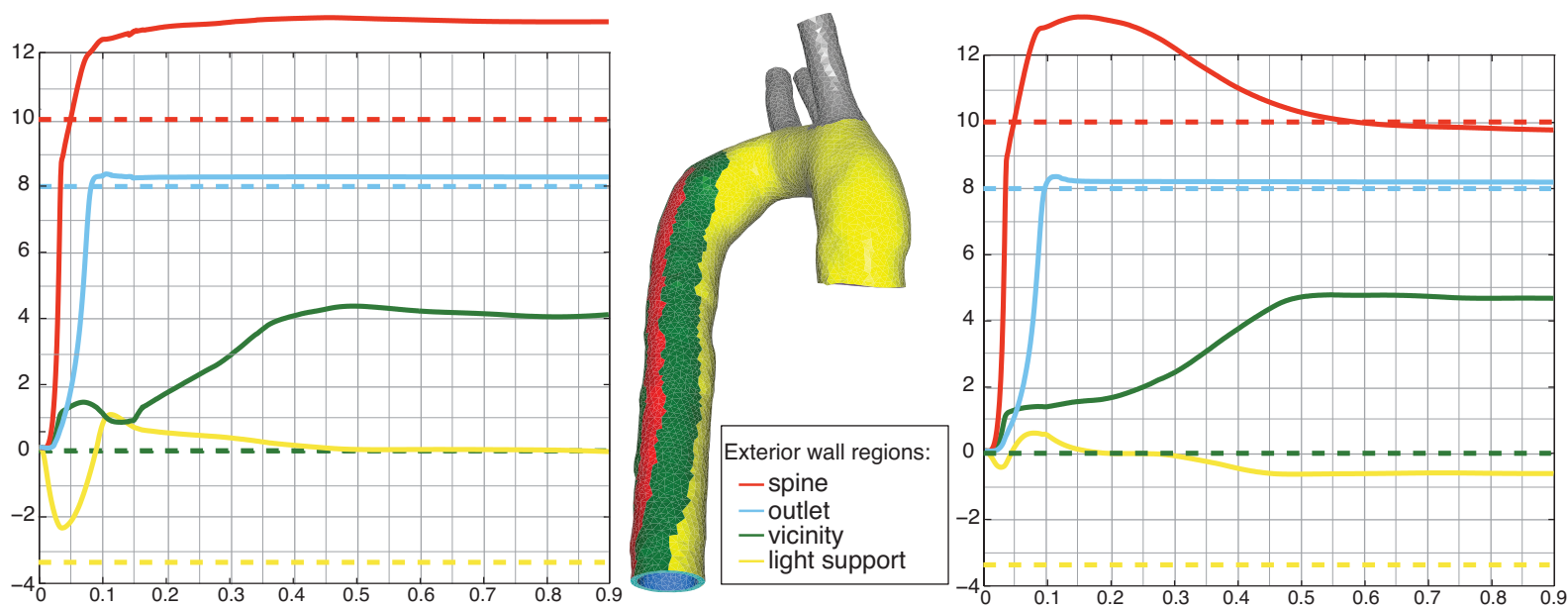

Fig. 15 Time identification of 4 parameters with synthetic data, data sampling $\Delta T=100 \delta t=0.1 T$ (left) and $\Delta T=10 \delta t=$ $0.01 T$ (right). Dashed lines: Target values; Solid lines: Estimated values

waveforms can be acquired non-invasively using ultrasound or phase-contrast magnetic resonance imaging. As already mentioned, these measurements can directly enter our estimation formalism on the fluid side - see Figure 5. Moreover, in addition to state variables and their directly derived quantities, one may have measurements of material parameters such as vessel wall thickness, stiffness or distensibility. In this regard, it should be noted that parameters are considered by extension as generalized state variables in the joint stateparameter estimation framework (Moireau et al 2008), hence these measurements can directly be used in the filtering corrections. However, the integration of certain types of observations would require further extensions of the proposed strategy, especially when corresponding to quantities not instantaneously associated with the current state and parameter variables at each observation time. For instance, in the case of blood pressure, when acquired non-invasively only the bounds of the state variable (i.e., systolic and diastolic pressures) are registered. Another example is given by pressure pulse velocity observations easily measurable non-invasively for the assessment of hypertension. This situation may preclude the estimation "on the fly" since usually a complete cycle of the solution should be produced in order to quantify the pulse velocity.

Simultaneous estimation of stiffness and external tissue support - In this article, we illustrated the application of a sequential framework based on filtering methods to the estimation of the material parameters defining the support provided by the different tissues and organs around a subject-specific model of the thoracic aorta. Here, a given value for the stiffness of the arterial wall was assumed to be known. In a previous investigation
(Bertoglio et al 2012b) the same framework was applied to the identification of the elastic properties of the arterial wall in an idealized fluid-structure interaction model, neglecting the support of external tissues and organs. The simultaneous estimation of arterial wall stiffness and external tissue support has therefore not been attempted thus far. This task will ultimately provide the most valuable information since it would give estimates on parameters that have been known to be predictors of disease (i.e., arterial stiffness) together with the parameters that enable the representation of realistic motion patterns of the arterial structures (i.e., the external tissue support).

In order to simultaneously estimate arterial stiffness and tissue support, richer information on the arterial kinematics is needed. This information could potentially be obtained using newer modalities of timeresolved medical image data to discriminate between the various components of the motion of the arterial tissue: namely, the deformation due to the oscillating pressure field, the motion resulting from respiration, and the cardiac motion. This would, indeed, enable the identifiability of arterial stiffness - via measurements related to pressure-induced contribution - distinctly from the tissue support properties, while cardiac and respiratory contributions would provide directly relevant information on the corresponding external supports. Notable progress has been made in cardiac and respiratory compensation in Magnetic Resonance Imaging using cardiac gated and respiratory navigators (Buerger et al 2011). Further improvements in image data acquisition techniques, together with the sequential estimation framework presented here will undoubtedly bring us closer to the goal of extracting physiologically meaningful mate- 
rial data from images. Lastly, it is important to note that the final goal of the framework sketched here is to provide estimates not only for the material properties of the arterial tissue, but also on the input and output flow boundary conditions, via proper integration of image data, pressure, flow, derived state quantities and discrete measurements of material parameters. It is through the simultaneous consideration of these various observations that this framework will be capable of automatically tuning the material parameters and inflow and outflow conditions required in the simulation process.

Algorithm effectiveness and estimation benefits - In Figure 13 we observed (1) compared to light tissue support, an improvement of the distances, in particular near the end of the time window and (2) compared to the manually calibrated tissue support a slight improvement all along the simulation. This improvement is further illustrated in Figure 14 where we can see that the contours of the simulation with the estimated values are significantly closer to the segmented contours than for the simulation with the values taken from (Moireau et al 2011). This final validation of the results of the estimation procedure - in particular in the "spine region" and at the outlet - justifies in itself the interest of such a sequential estimation methodology, especially when considering that this estimation procedure can be performed in a time comparable to a classical simulation. In (Moireau et al 2011), the interest of the external tissue support was already clearly established by comparing the distances obtained with external tissue support against the configuration where no tissue support was considered with only homogeneous Dirichlet conditions imposed at the inlet and outlets - recall Figure 9. However, in (Moireau et al 2011) this manual calibration performed required great care and multiple simulations to quantitatively set up the parameters, whereas here the procedure is fully automatic and produces comparatively better results.

The computational complexity of our algorithm should be compared to other state-of-the-art identification procedures. For example, let us consider a variational identification strategy where we minimize a criterion based on the discrepancy measure integrated over time plus additional regularization terms, as was used in the context of blood flows in (Martin et al 2005) with a very effective minimization algorithm. Even so, the complete procedure for one single parameter value in $1 D$ requires 6 to 10 iterations, each requiring a complete heart beat forward simulation and a backward simulation of the adjoint problem. The adjoint system - being linear is less intensive to solve, notwithstanding the difficulty of storing all the state solutions and discrepancies over time. Overall, the computational time-cost of the complete identification is then at least $10 \mathrm{~T}_{\text {ref }}$, meaning several days of computations on a standard workstation. Even worse, estimating multiple parameter values as in the present study would lead to many additional minimization iterations which increase the computational costs all the more. Here we recall for comparison that our estimation procedure CPU time is roughly $2 \mathrm{~T}_{\text {ref }}$ irrespective of the number of parameter values to be estimated.

\section{Concluding remarks}

We have proposed a complete methodological chain for the identification of boundary support parameters in a fluid-structure vascular model using patient image data, based on well-adapted sequential data assimilation procedures. We subsequently demonstrated the use of this framework in a real life application case in which we successfully performed the automatic characterization of the tethering to the spine and to the downstream vasculature, and most importantly identified the actual regions in which the boundary support modeling is necessary. The simulations performed with the estimated parameter values proved more accurate than with the previous expert manual calibration presented in (Moireau et al 2011). In this realistic example the estimation procedure itself was shown to be computationally effective - comparable to a direct simulation in terms of computation time, indeed - and robust, in particular with respect to parameter initial values.

This methodological framework paves the way for complete patient-specific vascular modeling, in which ultimately all the available measurements could be used to estimate the various uncertain parameters needed for the patient-specific simulations. Moreover, some of these parameters are in itself of utmost clinical value for diagnosis purposes, which represents an important additional motivation for estimation. Finally, estimation can also be envisioned as an integrated tool for investigating intrinsic modeling validity, since a lack of convergence of some estimated modeling parameters provided identifiability has been established by preliminary synthetic trials and measurement errors are adequately controlled - can only be accounted for by modeling shortcomings. This is especially valuable in the context of patient-specific modeling in which a priori testing cannot be used to assess the model validity. 


\section{References}

Alastrué V, Garcia A, Peña E, Rodríguez J, Martínez M, Doblaré M (2010) Numerical framework for patientspecific computational modelling of vascular tissue. Int J Numer Meth Biomed Engng 26(1):35-51

Auroux D, Blum J (2008) A nudging-based data assimilation method: the Back and Forth Nudging (BFN) algorithm. Nonlinear Processes Geophysics 15:305-319

Baerentzen J, Aanaes H (2005) Signed distance computation using the angle weighted pseudo-normal. IEEE Transactions on Visualization and Computer Graphics 11(3):243253

Bellman R (1957) Dynamic Programming. Princeton University Press

Bertoglio C, Chapelle D, Fernández M, Gerbeau JF, Moireau P (2012a) State observers of a vascular fluid-structure interaction model through measurements in the solid, submitted to CMAME

Bertoglio C, Moireau P, Gerbeau JF (2012b) Sequential parameter estimation for fluid-structure problems. application to hemodynamics. Int J Num Meth Biomedical Engng 28(4):434-455, DOI: $10.1002 / \mathrm{cnm} .1476$

Blum J, Le Dimet FX, Navon I (2009) Data assimilation for geophysical fluids. Computational Methods for the Atmosphere and the Oceans 14:385-441

Brown A, Shi Y, Arndt A, Müller J, Lawford P, Hose D (2011) Importance of realistic LVAD profiles for assisted aortic simulations: evaluation of optimal outflow anastomosis locations

Buerger C, Schaeffter T, King A (2011) Hierarchical adaptive local affine registration for fast and robust respiratory motion estimation. Medical Image Analysis 15(4):551-564

Caselles V, Kimmel R, Shapiro G (1997) Geodesic active contours. International Journal of Computer Vision 22(1):61-79

Cebral J, Castro M, Appanaboyina S, Putman C, Millan D, Frangi A (2005) Efficient pipeline for image-based patient-specific analysis of cerebral aneurysm hemodynamics: technique and sensitivity. IEEE transactions on medical imaging 24(4):457-467

Chabiniok R, Moireau P, Lesault PF, Rahmouni A, Deux JF, Chapelle D (2011) Estimation of tissue contractility from cardiac cine-MRI using a biomechanical heart model. Biomechanics and Modeling in Mechanobiology 11(5):609-630, DOI 10.1007/s10237-011-0337-8

Chapelle D, Cindea N, Moireau P (2012) Improving convergence in numerical analysis using observers. the wave-like equation case. M3AS In press

Chavent G (2010) Nonlinear Least Squares for Inverse Problems. No. XIV in Theoretical Foundations and Step-byStep Guide for Applications, Springer

Fernández M, Gerbeau JF, Grandmont C (2007) A projection semi-implicit scheme for the coupling of an elastic structure with an incompressible fluid. Internat J Numer Methods Engrg 69(4):794-821

Fernández M, Gerbeau JF (2009) Fluid structure interaction problems in haemodynamics, Springer Verlag, chap 9. In Mathematical Modelling of the Cardiovascular System, L. Formaggia, A. Quarteroni, A. Veneziani editors

Figueroa C, Vignon-Clementel I, Jansen K, Hughes T, Taylor C (2006) A coupled momentum method for modeling blood flow in three-dimensional deformable arteries. Comput Methods Appl Mech Engrg 195(41-43):5685-5706

Formaggia L, Gerbeau JF, Nobile F, Quarteroni A (2001) On the coupling of $3 \mathrm{D}$ and $1 \mathrm{D}$ Navier-Stokes equations for flow problems in compliant vessels. Comp Meth Appl Mech Engrg 191(6-7):561-582

Gee M, Förster C, Wall W (2010) A computational strategy for prestressing patient-specific biomechanical problems under finite deformation. International Journal for $\mathrm{Nu}$ merical Methods in Biomedical Engineering 26(1):52 - 72

Gerbeau JF, Vidrascu M, Frey P (2005) Fluid-structure interaction in blood flows on geometries based on medical imaging. Comp \& Struct 83(2-3):155-165

Julier S, Uhlmann J (2002) Reduced sigma point filters for the propagation of means and covariances through nonlinear transformations. In: Proc. of IEEE Am.Contr.Conf., pp 887-892

Kailath T, Sayed A, Hassibi B (2000) Linear estimation. Prentice-Hall, Inc.

Kerckhoffs R, Neal M, Gu Q, Bassingthwaighte J, Omens J, Mcculloch A (2007) Coupling of a 3D finite element model of cardiac ventricular mechanics to lumped systems models of the systemic and pulmonic circulation. Annals of Biomedical Engineering 35(1):1-18

Luenberger DG (1971) An introduction to observers. IEEE Transactions on Automatic Control 16:596-602

Martin V, Clément F, Decoene A, Gerbeau JF (2005) Parameter identification for a one-dimensional blood flow model. ESAIM-Proceedings 14:174-200, DOI 10.1051/ proc:2005014

Moireau P, Chapelle D (2011a) Erratum of article "reducedorder Unscented Kalman Filtering with application to parameter identification in large-dimensional systems". COCV 17:406-409, doi:10.1051/cocv/2011001

Moireau P, Chapelle D (2011b) Reduced-order Unscented Kalman Filtering with application to parameter identification in large-dimensional systems. Cont Optim and Calc Variat 17:380-405, DOI 10.1051/cocv/2010006

Moireau P, Chapelle D, Le Tallec P (2008) Joint state and parameter estimation for distributed mechanical systems. Computer Methods in Applied Mechanics and Engineering 197:659-677

Moireau P, Chapelle D, Le Tallec P (2009) Filtering for distributed mechanical systems using position measurements: Perspectives in medical imaging. Inverse Problems 25(3):035,010 (25pp)

Moireau P, Xiao N, Astorino M, Figueroa C, Chapelle D, Taylor C, Gerbeau JF (2011) External tissue support and fluid-structure simulation in blood flows. Biomechanics and Modeling in Mechanobiology 11(1):1-18, DOI 10.1007/s10237-011-0289-z

Moreno R, Nicoud F, Veunac L, Rousseau H (2006) Nonlinear-transformation-field to build moving meshes for patient specific blood flow simulations. In: Wesseling $\mathrm{P}$, Oñate E, Périaux J (eds) ECCOMAS CFD

Navon I (2009) Data assimilation for numerical weather prediction: A review. In: Data assimilation for atmospheric, oceanic, hydrologic applications, Springer

Perego M, Veneziani A, Vergara C (2011) A variational approach for estimating the compliance of the cardiovascular tissue: An inverse fluid-structure interaction problem. SIAM J Sc Comp 33(3):1181-1211

Peyré G, Cohen L (2008) Heuristically driven front propagation for fast geodesic extraction. International Journal for Computational Vision and Biomechanics 1(1):55-67

Pham DT (2001) Stochastic methods for sequential data assimilation in strongly nonlinear systems. Monthly weather review 129(5):1194-1207

Pham DT, Verron J, Gourdeau L (1998) Filtres de Kalman singuliers évolutifs pour l'assimilation de données en 
océanographie. Comptes Rendus de l'Académie des Sciences - Series IIA 326(4):255-260, DOI DOI:10.1016/ S1251-8050(97)86815-2

Piccinelli M, Mirabella L, Passerini T, Haber E, Veneziani A (2010) 4D image-based CFD simulation of a compliant blood vessel. Tech. Rep. TR-2010-027, Emory University

Quarteroni A, Ragni S, Veneziani A (2001) Coupling between lumped and distributed models for blood flow problems. Comput Vis Sci 4(2):111-124, second AMIF International Conference (Il Ciocco, 2000)

Simon D (2006) Optimal State Estimation: Kalman, $H^{\infty}$, and Nonlinear Approaches. Wiley-Interscience

Troianowski G, Taylor C, Feinstein J, Vignon-Clementel I (2011) Three-dimensional simulations in Glenn patients: clinically based boundary conditions, hemodynamic results and sensitivity to input data. Journal of Biomechanical Engineering 133:111,006

Vignon-Clementel I, Figueroa C, Jansen K, Taylor C (2010) Outflow boundary conditions for 3D simulations of nonperiodic blood flow and pressure fields in deformable arteries. Computer Methods in Biomechanics and Biomedical Engineering

Xi J, Lamata P, Lee J, Moireau P, Chapelle D, Smith N (2011) Myocardial transversely isotropic material parameter estimation from in-silico measurements based on reduced-order unscented Kalman filter. $J$ of the Mechanical Behavior of Biomedical Materials 4:1090-1102, doi:10.1016/j.jmbbm.2011.03.018

Xiong G, Figueroa C, Xiao N, Taylor C (2010) Simulation of blood flow in deformable vessels using subject-specific geometry and assigned variable mechanical wall properties. International Journal for Numerical Methods in Biomedical Engineering 27(7):1000-1016 